\title{
Resource-distribution via Boolean constraints
}

\author{
James Harland ${ }^{*}$ David Pym ${ }^{\dagger}$
}

November 11, 2018

\begin{abstract}
We consider the problem of searching for proofs in sequential presentations of logics with multiplicative (or intensional) connectives. Specifically, we start with the multiplicative fragment of linear logic and extend, on the one hand, to linear logic with its additives and, on the other, to the additives of the logic of bunched implications, BI. We give an algebraic method for calculating the distribution of the side-formulæ in multiplicative rules which allows the occurrence or non-occurrence of a formula on a branch of a proof to be determined once sufficient information is available. Each formula in the conclusion of such a rule is assigned a Boolean expression. As a search proceeds, a set of Boolean constraint equations is generated. We show that a solution to such a set of equations determines a proof corresponding to the given search. We explain a range of strategies, from the lazy to the eager, for solving sets of constraint equations. We indicate how to apply our methods systematically to large family of relevant systems.
\end{abstract}

\section{Introduction}

Proof-search in logics, such as linear logic (LL) [2] or the logic of bunched implications (BI) [8, 10, 11], which have multiplicative connectives requires a mechanism by which the distribution of formulæ, sometimes viewed as resources, between different branches of a proof may be calculated. Such mechanisms are usually specified by intricate rules of inference which are used to keep track of the relevant information [6, 17] and are tailored for one particular distribution mechanism.

We show how a judicious use of Boolean expressions admits an explicit statement of the resource distribution problem (unlike in the standard sequent calculus for such logics) without making a commitment to a particular strategy for managing the distribution (unlike the tailored inference systems of [6, 17]). Thus we pursue an algebraic approach to this particular problem of proof-search, allowing a separation of the specification of the problem from the method of its solution.

The key step in our approach is to assign a Boolean expression to each of the side-formulæ in multiplicative rules (i.e., just those formulæ which require distribution). Constraints on the possible values of this expression are then generated during the search process and propagated up the search tree, resulting in a set of Boolean equations. A successful proof-search will generate a solution to this set of equations and hence the corresponding distribution of formulæ across the multiplicative branches of the proof may be calculated.

We show how a range of different strategies for solving the Boolean constraints may be considered in this framework, whilst maintaining the same set of inference rules. Hence the algebraic approach allows us to separate the inference process from the precise distribution of formulæ across the branches.

To illustrate the process, consider the $\otimes \mathrm{R}$ and $\ngtr \mathrm{L}$ rules of linear logic [2] below.

$$
\frac{\Gamma_{1} \vdash \phi_{1}, \Delta_{1} \quad \Gamma_{2} \vdash \phi_{2}, \Delta_{2}}{\Gamma_{1}, \Gamma_{2} \vdash \phi_{1} \otimes \phi_{2}, \Delta_{1}, \Delta_{2}} \otimes \mathrm{R} \quad \text { and } \quad \frac{\Gamma_{1}, \phi_{1} \vdash \Delta_{1} \quad \Gamma_{2}, \phi_{2} \vdash \Delta_{2}}{\Gamma_{1}, \Gamma_{2}, \phi_{1} \diamond \phi_{2} \vdash \Delta_{1}, \Delta_{2}} 8 \mathrm{~L},
$$

When considered as rules of deductive inference (i.e., proofs are generated from premisses to conclusion), there is no distribution problem; the multisets of formulæ $\Gamma_{1}, \Gamma_{2}, \Delta_{1}$ and $\Delta_{2}$ are known in advance. However, when

\footnotetext{
${ }^{*}$ Department of Computer Science, Royal Melbourne Institute of Technology, GPO Box 2476V, Melbourne 3001, Australia. Email: jah@cs.rmit.edu.au; WWW: www.cs.rmit.edu.au/ jah

${ }^{\dagger}$ Department of Computer Science, Queen Mary, University of London, London E1 4NS, U.K. Email: pym@dcs.qmw.ac.uk; WWW: www.dcs.qmw.ac.uk/ pym
} 
considered as rules of search (i.e., reductive inference), then these four multisets are unknown and must be calculated in the course of proof-search.

In $\S 2$, we address this problem for multiplicative linear logic, MLL. In particular, we show how the problem of distributing the side-formulæ may be addressed by attaching a Boolean expression to each such formula together with some simple rules for the generation of constraints on the value of this expression. The problem of allocation of the formula to a branch is thus reduced to determining (in an appropriate manner) a solution to a set of equations over Boolean expressions. We show that this method is sound and complete for this logic.

In $\S 3$, we extend the results of the previous section to include the additive and exponential connectives of linear logic, i.e., to propositional linear logic, PLL. This is quite straightforward.

In $\S 4$, we extend the approach of $\S$ - 2 to the (propositional fragment of) the logic of bunched implications, BI [10]. This may be seen as a different extension of MLL, in that the additive connectives are introduced in a more intricate manner. Accordingly the results are correspondingly more subtle in this case.

In $\oint$, we discuss the manner in which the equations generated by the inference rules may be systematically collected and solved. There are three main classes of strategy here: lazy, eager and intermediate. Lazy strategies are those in which one branch of the proof-tree is selected and then followed to completion;the corresponding equations are solved and then the solution which has been found is broadcast to all other branches. Another branch is then selected, and the process continues. Such strategies have properties similar to depth-first search and are typically used in the implementation of linear logic programming languages such as Lygon [4, 17, 14] and Lolli [6]. Eager strategies are those in which all branches are searched in parallel and one single set of equations is collected. This single set of equations is then solved once all equations have been found. Intermediate strategies solve a fixed number of branches' worth of equations at one time. We discuss how each of these classes of strategies may be utilized in our framework.

In $\S$, we conclude with an account of the application of our methods to the family of relevant logics, as described systematically by Read [12].

We consider our approach to be an algebraic view of proof-search; in particular, this approach may be considered as a form of labelled deduction [1]. Our approach is similar in spirit to that of proof-nets [2] and has its origins in the notion of path introduced in [14]; however, it should be noted that in our case we recover multiplicative consequences from additive rules by the use of Boolean constraints, rather than recover additive consequences from an underlying multiplicative system. We have suggested that it is helpful to consider the formulæ to be distributed as resources and, indeed, in the case of linear logic there is a very simple sense in which this is possible: LL can be considered to count the number of occurrences of a formula. The treatment of resources provided by $\mathbf{B I}$, on the other hand, is more delicate. This point is discussed further in $\S$.

Some previous results on linear logic were presented in [5]; in the present paper we develop these results further and extend them to $\mathbf{B I}$.

\section{Multiplicatives}

We begin by restricting our attention to multiplicative linear logic $\mathbf{M L L}$; this is a fragment common to both linear logic and BI. In addition, throughout the paper we consider only propositional fragments. Quantifiers pose no problem per $s e$ and, especially in the case of linear logic, may be incorporated into what follows in a straightforward manner 1 However, the essential issues are more simply described in their absence.

Definition 1 The formula of (propositional) multiplicative linear logic MLL are as follows:

$$
p|\phi \otimes \phi| \phi 8 \phi|\phi \multimap \phi| \phi^{\perp}|\mathbf{1}| \perp
$$

where $p$ is an atom.

Note that we do not include the exponentials here; further discussion on this point may be found in $\$ 3$.

In order to represent the appropriate constraints on the distribution of formulæ, we do not require arbitrary Boolean expressions. In particular, we require only those given by the following grammar:

$$
e::=x|\bar{x}| x . e \mid \bar{x} . e
$$

\footnotetext{
${ }^{1}$ Note that quantifiers for linear logic were included in the earlier paper [ff].
} 
where $x$ is a Boolean variable.

However, the results of this paper are not dependent on the precise class of formulæ, and hence any class of Boolean expressions which includes those above will suffice. 4

Definition 2 An annotated formula is a formula $\phi$ together with a Boolean expression e, denoted as $\phi[e]$. We denote by $\exp (\phi)$ the Boolean expression associated with the annotated formula $\phi$. A sequent consisting entirely of annotated formula is known as a resource sequent.

In general, the state of the knowledge of the distribution of the formulæ is characterized by the state of knowledge of the Boolean variables, with the distribution of the formula known iff the corresponding Boolean expression has been assigned a value. Hence in addition to the proof tree, we maintain an assignment of the Boolean variables which appear in the proof tree.

Definition 3 Given a multiset of annotated formula $\Delta=\left\{\phi_{1}\left[e_{1}\right], \ldots \phi_{n}\left[e_{n}\right]\right\}$ and a total assignment I of the Boolean variables in $\Delta$, we define $\Delta[I]=\left\{\phi_{1}\left[v_{1}\right], \ldots \phi_{n}\left[v_{n}\right]\right\}$, where $e_{i}$ has the value $v_{i}$ under $I$. We denote by $\Delta[I]^{1}$ the multiset of annotated formula $\phi[e]$ in $\Delta[I]$ such that e evaluates to 1 under $I$.

We will often identify an unannotated formula $\phi$ with the annotated formula $\phi[1]$ (for instance, in Proposition 1); it will always be possible to disambiguate such annotations from the context.

Definition 4 Let $V=\left\{x_{1}, x_{2}, \ldots x_{n}\right\}$ be a set of Boolean variables. Then we denote by $\bar{V}$ the set of Boolean expressions $\left\{\overline{x_{1}}, \overline{x_{2}}, \ldots \overline{x_{n}}\right\}$. We denote by $\{e\}^{n}$ the multiset which contains $n$ copies of the Boolean expression $e$.

Definition 5 Let $\Gamma=\left\{\phi_{1}\left[e_{1}\right], \phi_{2}\left[e_{2}\right], \ldots \phi_{n}\left[e_{n}\right]\right\}$ be a multiset of annotated formula and let $\left\{x_{1}, x_{2}, \ldots, x_{n}\right\}$ be a set of Boolean variables not occurring in $\Gamma$. Then we define

$$
\Gamma .\left\{x_{1}, x_{2}, \ldots, x_{n}\right\}=\left\{\phi_{1}\left[e_{1} . x_{1}\right], \phi_{2}\left[e_{2} . x_{2}\right], \ldots, \phi_{n}\left[e_{n} . x_{n}\right]\right\} .
$$

We are now in a position to define the inference rules. Roughly speaking, the Boolean constraints are introduced by the rules which require multiplicative distribution of formulæ $(\otimes R, 8 \mathrm{~L},-\mathrm{L})$, are resolved by rules with no premisses (Axiom, $\perp \mathrm{L}, 1 \mathrm{R}$ ) and are maintained by all the others. Note also that the principal formula of each rule must be assigned the value 1 ; hence the selection of the principal formula also makes some contribution to the solution of the equations.

Definition 6 We define the following sequent calculus with constraints for MLL:

$$
\begin{aligned}
& \text { Axiom } \frac{e_{1}=e_{2}=1 \forall e_{3} \in \exp (\Gamma \cup \Delta) e_{3}=0}{\Gamma, p\left[e_{1}\right] \vdash p\left[e_{2}\right], \Delta} \\
& \perp \mathrm{L} \quad \frac{\forall e_{2} \in \exp (\Gamma \cup \Delta) e_{2}=0}{\Gamma, \perp\left[e_{1}\right] \vdash \Delta} e_{1}=1 \quad \perp \mathrm{R} \quad \frac{\Gamma \vdash \Delta}{\Gamma \vdash \perp[e], \Delta} e=1
\end{aligned}
$$

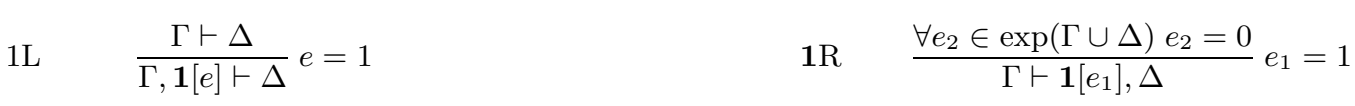

$$
\begin{aligned}
& \text { ৪L } \quad \frac{\Gamma \cdot V, \phi_{1}[e] \vdash \Delta . W \quad \Gamma \cdot \bar{V}, \phi_{2}[e] \vdash \Delta \cdot \bar{W}}{\Gamma,\left(\phi_{1} 8 \phi_{2}\right)[e] \vdash \Delta} e=1 \quad \text { \& } \quad \frac{\Gamma \vdash \phi_{1}[e], \phi_{2}[e], \Delta}{\Gamma \vdash\left(\phi_{1} 8 \phi_{2}\right)[e], \Delta} e=1 \\
& \otimes \mathrm{L} \quad \frac{\Gamma, \phi_{1}[e], \phi_{2}[e] \vdash \Delta}{\Gamma,\left(\phi_{1} \otimes \phi_{2}\right)[e] \vdash \Delta} e=1 \quad \otimes \mathrm{R} \quad \frac{\Gamma \cdot V \vdash \phi_{1}[e], \Delta . W \quad \Gamma \cdot \bar{V} \vdash \phi_{2}[e], \Delta \cdot \bar{W}}{\Gamma \vdash\left(\phi_{1} \otimes \phi_{2}\right)[e], \Delta} e=1 \\
& \multimap \mathrm{L} \quad \frac{\Gamma \cdot V \vdash \phi_{1}[e], \Delta . W \quad \Gamma \cdot \bar{V}, \phi_{2}[e] \vdash \Delta . \bar{W}}{\Gamma,\left(\phi_{1} \multimap \phi_{2}\right)[e] \vdash \Delta} e=1 \quad \multimap \mathrm{R} \quad \frac{\Gamma, \phi_{1}[e] \vdash \phi_{2}[e], \Delta}{\Gamma \vdash\left(\phi_{1} \multimap \phi_{2}\right)[e], \Delta} e=1 \\
& \mathrm{~L}^{\perp} \quad \frac{\Gamma \vdash \phi[e], \Delta}{\Gamma, \phi^{\perp}[e] \vdash \Delta} e=1 \quad \mathrm{R}^{\perp} \quad \frac{\Gamma, \phi[e] \vdash \Delta}{\Gamma \vdash \phi^{\perp}[e], \Delta} e=1
\end{aligned}
$$

\footnotetext{
${ }^{2}$ Naturally such considerations are vital when the complexity of determining the satisfiability of such expressions is concerned. However, this is a topic beyond the scope of the present paper.
} 
where the rules $\otimes \mathrm{R}, 8 \mathrm{~L}$ and $\multimap \mathrm{L}$ have the side-condition that $V$ and $W$ are disjoint sets of Boolean variables, none of which occur in $\Gamma, \Delta, \phi_{1}$ or $\phi_{2}$. We refer to the rules Axiom, $1 \mathrm{R}$ and $\perp \mathrm{L}$ as leaf rules.

We then require a proof in the above system to consist of not just the appropriate proof tree, but also an assignment of the Boolean variables which occur in it.

Definition 7 A resource derivation is a tree regulated by the rules of the resource calculus in which each formula of the endsequent is assigned a distinct Boolean variable, together with a partial assignment of the Boolean variables appearing in the derivation.

A resource derivation is total if its assignment of the Boolean variables is total. Otherwise, the resource derivation is partial. A resource derivation is closed if all of the leaves of the proof tree are instances of one of the leaf rules. $A$ resource proof is a total, closed resource derivation in which all the Boolean variables in the endsequent and all principal formula are assigned the value 1.

For notational simplicity, a lack of annotation in any of the rules of the resource calculus implies that the constraints currently applicable to the formula are not changed.

Given a resource derivation, it is then straightforward to recover the corresponding linear proof tree. Note that when the resource derivation is a resource proof, this linear proof tree will actually be a proof in the linear sequent calculus.

Definition 8 Let $R$ be a total resource derivation, with proof tree $T$ and Boolean assignment $I$. The linear proof tree corresponding to $\mathrm{R}$ under $\mathrm{I}$ is the proof tree obtained by deleting from $T$ all formula whose Boolean expression evaluates to 0 under $I$.

There may be many resource proofs which have the same corresponding linear proof - for example, the linear proof corresponding to a resource proof of $\Gamma \vdash \Delta$ will be the same as one corresponding to $\Gamma, \phi[0] \vdash \Delta$ (see Lemma11).

Note that resource proofs do not alter the applicability of the inference rules. In particular, they do not allow us to construct a "proof" of $p \otimes q \vdash p \otimes q$ in which the right-hand tensor is reduced first: such a proof is prohibited by the requirement of the $\otimes \mathrm{L}$ rule that each of $p, q$ and $p \otimes q$ be assigned the same value.

To see this, consider the attempt below at such a resource proof:

$$
\frac{\frac{p[x . z], q[x . z] \vdash p[y]}{(p \otimes q)[x] \vdash p[y]} \otimes \mathrm{L} \quad \frac{p[x . \bar{z}], q[x . \bar{z}] \vdash q[y]}{(p \otimes q)[x . \bar{z}] \vdash q[y]}}{(p \otimes q)[x] \vdash(p \otimes q)[y]} \otimes \mathrm{L}
$$

Clearly we require that $x=1$ and $y=1$ in order for the rules to be applied, but but it is impossible to make either leaf of this tree into an instance of the Axiom rule, due to the impossibility of finding an appropriate value for $z$.

Resource-proofs are sound and complete with respect to MLL proofs. As usual, soundness reduces to showing that our global conditions are strong enough to recover proofs from the locally unsound system.

Proposition 1 (soundness of resource proofs) Let $\Gamma \vdash \Delta$ be a resource sequent in MLL. If $\Gamma \vdash \Delta$ has a resource proof $R$ with Boolean assignment $I$, then the linear proof tree corresponding to $R$ is a linear proof of $\Gamma[I]^{1} \vdash \Delta[I]^{1}$.

Proof By induction on the structure of resource proofs. Assume that $\Gamma \vdash \Delta$ has a resource proof.

In the base case, the sequent is the conclusion of either the Axiom, $\perp \mathrm{L}$ or $1 \mathrm{R}$ rules.

Axiom: We have $\Gamma, p\left[e_{1}\right] \vdash p\left[e_{2}\right], \Delta$ where $e_{1}=e_{2}=1$ and $\forall e_{3} \in \exp (\Gamma \cup \Delta) e_{3}=0$. Let this assignment of Boolean variables be $I$. Then $\Gamma[I]^{1},\left(p\left[e_{1}\right]\right)[I]^{1} \vdash\left(p\left[e_{2}\right]\right)[I]^{1}, \Delta[I]^{1}$ is just $p \vdash p$, and clearly the linear proof tree corresponding to $R$ is a proof of this sequent.

$\perp \mathrm{L}$ : We have $\Gamma, \perp\left[e_{1}\right] \vdash \Delta$ where $e_{1}=1$ and $\forall e_{2} \in \exp (\Gamma \cup \Delta) e_{2}=0$. Let this assignment of Boolean variables be $I$. Then $\Gamma[I]^{1},\left(\perp\left[e_{1}\right]\right)[I]^{1} \vdash \Delta[I]^{1}$ is just $\perp \vdash$, and clearly the linear proof tree corresponding to $R$ is a proof of this sequent.

1R: We have $\Gamma \vdash \mathbf{1}\left[e_{1}\right], \Delta$ where $e_{1}=1$ and $\forall e_{2} \in \exp (\Gamma \cup \Delta) e_{2}=0$. Let this assignment of Boolean variables be $I$. Then $\Gamma[I]^{1} \vdash\left(\mathbf{1}\left[e_{1}\right]\right)[I]^{1}, \Delta[I]^{1}$ is just $\vdash \mathbf{1}$, and clearly the linear proof tree corresponding to $R$ is a proof of this sequent. 
Hence we assume that the result holds for all provable sequents whose resource proof is no more than a given size.

Consider the last rule used in the proof. There are 10 cases:

1L: In this case, we have that $\Gamma=\Gamma^{\prime}, \mathbf{1}[e]$ where $e=1$, and there is a resource proof of $\Gamma^{\prime} \vdash \Delta$. By the hypothesis, there is a linear proof of $\Gamma^{\prime}[I]^{1} \vdash \Delta[I]^{1}$ for some Boolean assignment $I$, and hence there is a linear proof of $\Gamma^{\prime}[I]^{1}, \mathbf{1}[1] \vdash \Delta[I]^{1}$, which is just $\Gamma[I]^{1} \vdash \Delta[I]^{1}$, as required.

$\perp \mathrm{R}$ : In this case, we have that $\Delta=\perp[e], \Delta^{\prime}$ where $e=1$, and there is a resource proof of $\Gamma \vdash \Delta^{\prime}$. By the hypothesis, there is a linear proof of $\Gamma[I]^{1} \vdash \Delta^{\prime}[I]^{1}$ for some Boolean assignment $I$, and hence there is a linear proof of $\Gamma[I]^{1} \vdash \perp[1], \Delta^{\prime}[I]^{1}$, which is just $\Gamma[I]^{1} \vdash \Delta[I]^{1}$, as required.

8L: In this case, we have that $\Gamma=\Gamma^{\prime},\left(\phi_{1} 8 \phi_{2}\right)[e]$ where $e=1$, and $\Gamma^{\prime} . V, \phi_{1} \vdash \Delta . W$ and $\Gamma^{\prime} \cdot \bar{V}, \phi_{2} \vdash \Delta . \bar{W}$ both have resource proofs for some disjoint sets of Boolean variables $V$ and $W$. By the hypothesis, we have that there are linear proofs of $\left(\Gamma^{\prime} . V\right)[I]^{1},\left(\phi_{1}\right)[I]^{1} \vdash(\Delta . W)[I]^{1}$ and $\left(\Gamma^{\prime} \cdot \bar{V}\right)[I]^{1},\left(\phi_{2}\right)[I]^{1} \vdash(\Delta . \bar{W})[I]^{1}$ (recall that $I$ must be an assignment of all Boolean variables in the proof), and so there is a linear proof of $\left(\Gamma^{\prime} . V\right)[I]^{1},(\Gamma ; \bar{V})[I]^{1}\left(\phi_{1} \gamma^{8} \phi_{2}\right)[I]^{1} \vdash(\Delta . W)[I]^{1},(\Delta . \bar{W})[I]^{1}$ which is just $\Gamma[I]^{1} \vdash \Delta[I]^{1}$, as required.

४ R: In this case, we have that $\Delta=\left(\phi_{1} \ngtr \phi_{2}\right)[e], \Delta^{\prime}$ where $e=1$, and there is a resource proof of $\Gamma \vdash \phi_{1}, \phi_{2}, \Delta^{\prime}$. By the hypothesis, there is a linear proof of $\Gamma[I]^{1} \vdash \phi_{1}[I]^{1}, \phi_{2}[I]^{1}, \Delta^{\prime}[I]^{1}$ for some Boolean assignment $I$, and hence there is a linear proof of $\Gamma[I]^{1} \vdash\left(\phi_{1} 8 \phi_{2}\right)[I]^{1}, \Delta^{\prime}[I]^{1}$, which is just $\Gamma[I]^{1} \vdash \Delta[I]^{1}$, as required.

$\otimes \mathrm{L}:$ In this case, we have that $\Gamma=\left(\phi_{1} \otimes \phi_{2}\right)[e], \Gamma^{\prime}$ where $e=1$, and there is a resource proof of $\Gamma^{\prime}, \phi_{1}, \phi_{2} \vdash \Delta$. By the hypothesis, there is a linear proof of $\Gamma^{\prime}[I]^{1}, \phi_{1}[I]^{1}, \phi_{2}[I]^{1} \vdash \Delta[I]^{1}$ for some Boolean assignment $I$, and hence there is a linear proof of $\Gamma^{\prime}[I]^{1}\left(\phi_{1} \otimes \phi_{2}\right)[I]^{1} \vdash \Delta[I]^{1}$, which is just $\Gamma^{\prime}[I]^{1} \vdash \Delta[I]^{1}$, as required.

$\otimes \mathrm{R}:$ In this case, we have that $\Delta=\left(\phi_{1} \otimes \phi_{2}\right)[e], \Delta^{\prime}$ where $e=1$, and $\Gamma . V \vdash \phi_{1}, \Delta^{\prime} . W$ and $\Gamma \cdot \bar{V} \vdash \phi_{2}, \Delta^{\prime} \cdot \bar{W}$ both have resource proofs for some disjoint sets of Boolean variables $V$ and $W$. By the hypothesis, we have that there are linear proofs of $(\Gamma . V)[I]^{1} \vdash\left(\phi_{1}\right)[I]^{1},\left(\Delta^{\prime} . W\right)[I]^{1}$ and $(\Gamma \cdot \bar{V})[I]^{1} \vdash\left(\phi_{2}\right)[I]^{1},\left(\Delta^{\prime} . \bar{W}\right)[I]^{1}$ (recall that $I$ must be an assignment of all Boolean variables in the proof), and so there is a linear proof of $(\Gamma . V)[I]^{1},(\Gamma . \bar{V})[I]^{1} \vdash\left(\phi_{1} \otimes \phi_{2}\right)[I]^{1},\left(\Delta^{\prime} . W\right)[I]^{1},\left(\Delta^{\prime} . \bar{W}\right)[I]^{1}$ which is just $\Gamma[I]^{1} \vdash \Delta[I]^{1}$, as required.

$\multimap \mathrm{L}:$ In this case, we have that $\Gamma=\left(\phi_{1} \multimap \phi_{2}\right)[e 1], \Gamma^{\prime}$ where $e=1$, and $\Gamma^{\prime} . V \vdash \phi_{1}, \Delta . W$ and $\Gamma^{\prime} . \bar{V}, \phi_{2} \vdash \Delta . \bar{W}$ both have resource proofs for some disjoint sets of Boolean variables $V$ and $W$. By the hypothesis, we have that there are linear proofs of $\left(\Gamma^{\prime} . V\right)[I]^{1} \vdash\left(\phi_{1}\right)[I]^{1},(\Delta . W)[I]^{1}$ and $\left(\Gamma^{\prime} \cdot \bar{V}\right)[I]^{1},\left(\phi_{2}\right)[I]^{1} \vdash(\Delta . \bar{W})[I]^{1}$ (recall that $I$ must be an assignment of all Boolean variables in the proof), and so there is a linear proof of $\left(\Gamma^{\prime} . V\right)[I]^{1},\left(\Gamma^{\prime} \cdot \bar{V}\right)[I]^{1}\left(\phi_{1} \multimap \phi_{2}\right)[I]^{1} \vdash(\Delta . W)[I]^{1},(\Delta . \bar{W})[I]^{1}$ which is just $\Gamma[I]^{1} \vdash \Delta[I]^{1}$, as required.

$\multimap \mathrm{R}$ : In this case, we have that $\Delta=\left(\phi_{1} \multimap \phi_{2}\right)[e], \Delta^{\prime}$ where $e=1$, and there is a resource proof of $\Gamma, \phi_{1} \vdash$ $\phi_{2}, \Delta^{\prime}$. By the hypothesis, there is a linear proof of $\Gamma[I]^{1}, \phi_{1}[I]^{1} \vdash \phi_{2}[I]^{1}, \Delta^{\prime}[I]^{1}$ for some Boolean assignment $I$, and hence there is a linear proof of $\Gamma[I]^{1} \vdash\left(\phi_{1} \multimap \phi_{2}\right)[I]^{1}, \Delta^{\prime}[I]^{1}$, which is just $\Gamma[I]^{1} \vdash$ $\Delta[I]^{1}$, as required.

$L^{\perp}$ : In this case, we have that $\Gamma=\left(\phi^{\perp}\right)[e], \Gamma^{\prime}$ where $e=1$, and there is a resource proof of $\Gamma^{\prime} \vdash \phi, \Delta$. By the hypothesis, there is a linear proof of $\Gamma^{\prime}[I]^{1} \vdash \phi[I]^{1}, \Delta[I]^{1}$ for some Boolean assignment $I$, and hence there is a linear proof of $\Gamma^{\prime}[I]^{1},\left(\phi^{\perp}\right)[I]^{1} \vdash \Delta[I]^{1}$, which is just $\Gamma[I]^{1} \vdash \Delta[I]^{1}$, as required.

$R^{\perp}$ : In this case, we have that $\Delta=\left(\phi^{\perp}\right)[e], \Delta^{\prime}$ where $e=1$, and there is a resource proof of $\Gamma, \phi \vdash \Delta^{\prime}$. By the hypothesis, there is a linear proof of $\Gamma[I]^{1}, \phi[I]^{1} \vdash \Delta^{\prime}[I]^{1}$ for some Boolean assignment $I$, and hence there is a linear proof of $\Gamma[I]^{1} \vdash\left(\phi^{\perp}\right)[I]^{1}, \Delta^{\prime}[I]^{1}$, which is just $\Gamma[I]^{1} \vdash \Delta[I]^{1}$, as required.

In order to show the completeness of resource proofs, we require the following simple lemma:

Lemma 1 Let $\Gamma \vdash \Delta$ be a resource sequent in MLL. If $\Gamma \vdash \Delta$ has a closed resource derivation $R$, then $\Gamma, \phi[0] \vdash \Delta$ and $\Gamma \vdash \phi[0], \Delta$ also have closed resource derivations $R_{1}$ and $R_{2}$ respectively. Moreover, for any total assignment $I$ of the Boolean variables in $R$, the linear proof tree corresponding to $R$ under $I$ is the same as the linear proof trees corresponding to $R_{1}$ and $R_{2}$ respectively under $I$. 
Proof By induction on the structure of resource derivations. Assume that $\Gamma \vdash \Delta$ has a closed resource derivation.

In the base case, the sequent is the conclusion of either the Axiom, $\perp \mathrm{L}$ or $1 \mathrm{R}$ rules, and it is clear that the addition of $\phi[0]$ to either the antecedent or the succedent of $\Gamma \vdash \Delta$ will result in a closed resource derivation of the appropriate sequent. Clearly the linear proof tree property is satisfied in these cases.

Hence we assume that the result holds for all provable sequents whose resource proof is no more than a given size.

Consider the last rule used in the proof. There are 10 cases, of which we only give the argument for $\otimes \mathrm{L}$ and $\otimes R$; the others are similar.

$\otimes \mathbf{L}:$ In this case, we have that $\Gamma=\left(\phi_{1} \otimes \phi_{2}\right)[e], \Gamma^{\prime}$ where $e=1$, and there is a closed resource derivation of $\Gamma^{\prime}, \phi_{1}[1], \phi_{2}[1] \vdash \Delta$. By the hypothesis, there are closed resource derivations of both $\Gamma^{\prime}, \phi_{1}[1], \phi_{2}[1], \phi[0] \vdash$ $\Delta$ and $\Gamma^{\prime}, \phi_{1}[1], \phi_{2}[1] \vdash \Delta, \phi[0]$, and so there are closed resource derivations of $\Gamma^{\prime},\left(\phi_{1} \otimes \phi_{2}\right)[1], \phi[0] \vdash \Delta$ and $\Gamma^{\prime},\left(\phi_{1} \otimes \phi_{2}\right)[1] \vdash \Delta, \phi[0]$, as required.

Clearly the linear proof tree property is satisfied in this case.

$\otimes \mathrm{R}$ : In this case, we have that $\Delta=\left(\phi_{1} \otimes \phi_{2}\right)[e], \Delta^{\prime}$ where $e=1$, and $\Gamma . V \vdash \phi_{1}[1], \Delta^{\prime} . W$ and $\Gamma . \bar{V} \vdash$ $\phi_{2}[1], \Delta^{\prime} . \bar{W}$ both have closed resource derivations for some disjoint sets of Boolean variables $V$ and $W$. By the hypothesis, there are closed resource derivations of both $\Gamma . V, \phi[0] \vdash \phi_{1}[1], \Delta^{\prime} . W$ and $\Gamma . \bar{V}, \phi[0] \vdash$ $\phi_{2}[1], \Delta^{\prime} . \bar{W}$ and of both $\Gamma . V \vdash \phi_{1}[1], \phi[0], \Delta^{\prime} . W$ and $\Gamma . \bar{V} \vdash \phi_{2}[1], \phi[0], \Delta^{\prime} . \bar{W}$. Hence there are disjoint sets of variables $V^{\prime}$ and $W^{\prime}$ such that $V \subset V^{\prime}$ and $W \subset W^{\prime}$ such that $(\Gamma \cup \phi[0]) . V^{\prime} \vdash \phi_{1}[1], \Delta^{\prime} . W$ and $(\Gamma \cup \phi[0]) \cdot \overline{V^{\prime}} \vdash \phi_{2}[1], \Delta^{\prime} . \bar{W}$ both have closed resource derivations, as do $\Gamma . V \vdash \phi_{1}[1],\left(\Delta^{\prime} \cup \phi[0]\right) . W^{\prime}$ and $\Gamma \cdot \bar{V} \vdash \phi_{2}[1],\left(\Delta^{\prime} \cup \phi[0]\right) . \overline{W^{\prime}}$. Thus we have closed resource derivations of both $\Gamma, \phi[0] \vdash\left(\phi_{1} \otimes \phi_{2}\right)[1], \Delta^{\prime}$ and $\Gamma \vdash\left(\phi_{1} \otimes \phi_{2}\right)[1], \Delta^{\prime}, \phi[0]$, as required.

Clearly the linear proof tree property is satisfied in this case.

We are now in a position to show the completeness of resource proofs for MLL.

Proposition 2 (completeness of resource proofs) Let $\Gamma \vdash \Delta$ be a sequent in MLL. If $\Gamma \vdash \Delta$ has a proof $\Phi$ in MLL, then there are disjoint sets of Boolean variables $V$ and $W$ such that $\Gamma . V \vdash \Delta . W$ has a resource proof $R$ and the linear proof tree corresponding to $R$ is $\Phi$.

Proof By induction on the structure of resource proofs. Assume that $\Gamma \vdash \Delta$ has a resource proof.

In the base case, the sequent is the conclusion of either the Axiom, $\perp \mathrm{L}$ or $1 \mathrm{R}$ rules.

Axiom: We have $p \vdash p$, so clearly there is a resource proof of $p[x] \vdash p[y]$ and the linear proof corresponding to this resource proof is $\Phi$.

$\perp \mathrm{L}$ : We have $\perp \vdash$, so clearly there is a resource proof of $\perp[x] \vdash$ and the linear proof corresponding to this resource proof is $\Phi$.

1R: We have $\vdash \mathbf{1}$, so clearly there is a resource proof of $\vdash \mathbf{1}[x]$ and the linear proof corresponding to this resource proof is $\Phi$.

Hence we assume that the result holds for all provable sequents whose resource proof is no more than a given size.

Consider the last rule used in the proof. There are ten cases.

1L: In this case, we have that $\Gamma=\Gamma^{\prime}, \mathbf{1}$, and there is a proof of $\Gamma^{\prime} \vdash \Delta$ which is a subproof of $\Phi$. By the hypothesis, there are disjoint sets of variables $V$ and $W$ such that there is a resource proof of $\Gamma^{\prime} . V \vdash \Delta . W$, and hence there is a resource proof of $\Gamma^{\prime} . V, \mathbf{1}[1] \vdash \Delta . W$, and clearly the linear proof corresponding to this resource proof is $\Phi$. 
$\perp \mathrm{R}$ : In this case, we have that $\Delta=\Delta^{\prime}, \perp$, and there is a proof of $\Gamma \vdash \Delta^{\prime}$ which is a subproof of $\Phi$. By the hypothesis, there are disjoint sets of variables $V$ and $W$ such that there is a resource proof of $\Gamma . V \vdash \Delta^{\prime} . W$, and hence there is a resource proof of $\Gamma^{\prime} . V \vdash \perp[1], \Delta . W$, and clearly the linear proof corresponding to this resource proof is $\Phi$.

8L: In this case, we have that $\Gamma=\phi_{1} 8 \phi_{2}, \Gamma_{1} \Gamma_{2}, \Delta=\Delta_{1}, \Delta_{2}$ such that $\Gamma_{1}, \phi_{1} \vdash \Delta_{1}$ and $\Gamma_{2}, \phi_{2} \vdash$ $\Delta_{2}$ both have proofs in the linear sequent calculus which are subproofs of $\Phi$. Hence by the hypothesis there are disjoint sets of Boolean variables $V_{1}, V_{2}, W_{1}, W_{2}$ such that $\Gamma_{1} . V_{1}, \phi_{1}[1] \vdash \Delta_{1} . W_{1}$ and $\Gamma_{2} . V_{2}, \phi_{2}[1] \vdash \Delta_{2} . W_{2}$ have resource proofs, (and moreover the linear proofs corresponding to each resource proof is the appropriate subproof of $\Phi$ ) and so by Lemma 1 , there are closed resource derivations of $\Gamma_{1} \cdot V_{1}, \Gamma_{2} \cdot\{0\}^{n}, \phi_{1}[1] \vdash \Delta_{1} \cdot W_{1}, \Delta_{2} .\{0\}^{n}$ and $\Gamma_{1} \cdot\{0\}^{n}, \Gamma_{2} \cdot V_{2}, \phi_{2}[1] \vdash \Delta_{1} \cdot\{0\}^{n}, \Delta_{2} . W_{2}$. Hence there are new disjoint sets of Boolean variables (i.e., not occurring anywhere in the above two resource sequents) $V$ and $W$ and a total assignment $I$ of $V \cup W$ such that $\left(\Gamma_{1} \cdot V_{1}, \Gamma_{2} \cdot V_{2}\right) . V, \phi_{1}[1] \vdash\left(\Delta_{1} \cdot W_{1}, \Delta_{2} . W_{2}\right) . W$ and $\left(\Gamma_{1} \cdot V_{1}, \Gamma_{2} \cdot V_{2}\right) \cdot \bar{V}, \phi_{2}[1] \vdash\left(\Delta_{1} . W_{1}, \Delta_{2} \cdot W_{2}\right) \cdot \bar{W}$ have resource proofs, and so there is a resource proof of $\Gamma_{1} . V_{1}, \Gamma_{2} . V_{2},\left(\phi_{1} 8 \phi_{2}\right)[1] \vdash \Delta_{1} . W_{1}, \Delta_{2} . W_{2}$, i.e., $\Gamma . V^{\prime},\left(\phi_{1} \ngtr \phi_{2}\right)[1] \vdash \Delta . W^{\prime}$ for some disjoint sets of Boolean variables $V^{\prime}$ and $W^{\prime}$, and clearly the linear proof corresponding to this resource proof is $\Phi$.

8R: In this case, we have that $\Delta=\phi_{1} \ngtr \phi_{2}, \Delta^{\prime}$, and there is a proof of $\Gamma \vdash \phi_{1}, \phi_{2}, \Delta^{\prime}$ which is a subproof of $\Phi$. By the hypothesis, there are disjoint sets of variables $V$ and $W$ such that there is a resource proof of $\Gamma . V \vdash \phi_{1}[1], \phi_{2}[1], \Delta^{\prime} . W$, and hence there is a resource proof of $\Gamma^{\prime} . V \vdash\left(\phi_{1} 8 \phi_{2}\right)[1], \Delta . W$, and clearly the linear proof corresponding to this resource-proof is $\Phi$.

$\otimes \mathrm{L}$ : In this case, we have that $\Gamma=\phi_{1} \otimes \phi_{2}, \Gamma^{\prime}$, and there is a proof of $\Gamma^{\prime}, \phi_{1}, \phi_{2} \vdash \Delta$ which is a subproof of $\Phi$. By the hypothesis, there are disjoint sets of variables $V$ and $W$ such that there is a resource proof of $\Gamma^{\prime} . V, \phi_{1}[1], \phi_{2}[1] \vdash \Delta . W$, and hence there is a resource proof of $\Gamma^{\prime} . V,\left(\phi_{1} \otimes \phi_{2}\right)[1] \vdash \Delta . W$, and clearly the linear proof corresponding to this resource proof is $\Phi$.

$\otimes R$ : In this case, we have that $\Gamma=\Gamma_{1}, \Gamma_{2}, \Delta=\phi_{1} \otimes \phi_{2}, \Delta_{1}, \Delta_{2}$ such that $\Gamma_{1} \vdash \phi_{1}, \Delta_{1}$ and $\Gamma_{2} \vdash \phi_{2}, \Delta_{2}$ both have proofs in the linear sequent calculus which are subproofs of $\Phi$. Hence by the hypothesis there are disjoint sets of Boolean variables $V_{1}, V_{2}, W_{1}, W_{2}$ such that $\Gamma_{1} . V_{1} \vdash \phi_{1}[1], \Delta_{1} . W_{1}$ and $\Gamma_{2} . V_{2} \vdash \phi_{2}[1], \Delta_{2} . W_{2}$ have resource proofs, (and moreover the linear proofs corresponding to each resource proof is the appropriate subproof of $\Phi$ ) and so by Lemma 1 , there are closed resource derivations of $\Gamma_{1} \cdot V_{1}, \Gamma_{2} \cdot\{0\}^{n} \vdash$ $\phi_{1}[1], \Delta_{1} . W_{1}, \Delta_{2} .\{0\}^{n}$ and $\Gamma_{1} .\{0\}^{n}, \Gamma_{2} . V_{2} \vdash \phi_{2}[1], \Delta_{1} .\{0\}^{n}, \Delta_{2} . W_{2}$. Hence there are new disjoint sets of Boolean variables (i.e., not occurring anywhere in the above two resource sequents) $V$ and $W$ and a total assignment $I$ of $V \cup W$ such that $\left(\Gamma_{1} . V_{1}, \Gamma_{2} . V_{2}\right) . V \vdash \phi_{1}[1],\left(\Delta_{1} . W_{1}, \Delta_{2} . W_{2}\right) . W$ and $\left(\Gamma_{1} . V_{1}, \Gamma_{2} . V_{2}\right) \cdot \bar{V} \vdash$ $\phi_{2}[1],\left(\Delta_{1} . W_{1}, \Delta_{2} . W_{2}\right) \cdot \bar{W}$ have resource proofs, and so there is a resource proof of $\Gamma_{1} \cdot V_{1}, \Gamma_{2} \cdot V_{2} \vdash\left(\phi_{1} \otimes\right.$ $\left.\phi_{2}\right)[1], \Delta_{1} . W_{1}, \Delta_{2} . W_{2}$, i.e., $\Gamma . V^{\prime} \vdash\left(\phi_{1} \otimes \phi_{2}\right)[1], \Delta . W^{\prime}$ for some disjoint sets of Boolean variables $V^{\prime}$ and $W^{\prime}$, and clearly the linear proof corresponding to this resource proof is $\Phi$.

$\multimap \mathrm{L}$ : In this case, we have that $\Gamma=\phi_{1} \multimap \phi_{2}, \Gamma_{1}, \Gamma_{2}, \Delta=\Delta_{1}, \Delta_{2}$ such that $\Gamma_{1} \vdash \phi_{1}, \Delta_{1}$ and $\Gamma_{2}, \phi_{2} \vdash$ $\Delta_{2}$ both have proofs in the linear sequent calculus which are subproofs of $\Phi$. Hence by the hypothesis there are disjoint sets of Boolean variables $V_{1}, V_{2}, W_{1}, W_{2}$ such that $\Gamma_{1} . V_{1} \vdash \phi_{1}[1], \Delta_{1} . W_{1}$ and $\Gamma_{2} . V_{2}, \phi_{2}[1] \vdash \Delta_{2} . W_{2}$ have resource proofs, (and moreover the linear proofs corresponding to each resource proof is the appropriate subproof of $\Phi$ ) and so by Lemma 1 , there are closed resource derivations of $\Gamma_{1} . V_{1}, \Gamma_{2} \cdot\{0\}^{n} \vdash \phi_{1}[1], \Delta_{1} . W_{1}, \Delta_{2} .\{0\}^{n}$ and $\Gamma_{1} \cdot\{0\}^{n}, \Gamma_{2} . V_{2}, \phi_{2}[1] \vdash \Delta_{1} \cdot\{0\}^{n}, \Delta_{2} . W_{2}$. Hence there are new disjoint sets of Boolean variables (i.e., not occurring anywhere in the above two resource sequents) $V$ and $W$ and a total assignment $I$ of $V \cup W$ such that $\left(\Gamma_{1} \cdot V_{1}, \Gamma_{2} \cdot V_{2}\right) . V \vdash \phi_{1}[1],\left(\Delta_{1} \cdot W_{1}, \Delta_{2} \cdot W_{2}\right) . W$ and $\left(\Gamma_{1} \cdot V_{1}, \Gamma_{2} . V_{2}\right) \cdot \bar{V}, \phi_{2}[1] \vdash\left(\Delta_{1} . W_{1}, \Delta_{2} . W_{2}\right) \cdot \bar{W}$ have resource proofs, and so there is a resource proof of $\Gamma_{1} . V_{1}, \Gamma_{2} . V_{2},\left(\phi_{1} \multimap \phi_{2}\right)[1] \vdash \Delta_{1} . W_{1}, \Delta_{2} . W_{2}$, i.e., $\Gamma . V^{\prime},\left(\phi_{1} \multimap \phi_{2}\right)[1] \vdash \Delta . W^{\prime}$ for some disjoint sets of Boolean variables $V^{\prime}$ and $W^{\prime}$, and clearly the linear proof corresponding to this resource proof is $\Phi$.

$\multimap \mathrm{R}$ : In this case, we have that $\Delta=\phi_{1} \multimap \phi_{2}, \Delta^{\prime}$, and there is a proof of $\Gamma, \phi_{1} \vdash \phi_{2}, \Delta^{\prime}$ which is a subproof of $\Phi$. By the hypothesis, there are disjoint sets of variables $V$ and $W$ such that there is a resource proof of $\Gamma . V, \phi_{1}[1] \vdash \phi_{2}[1], \Delta^{\prime} . W$, and hence there is a resource proof of $\Gamma^{\prime} . V \vdash\left(\phi_{1} \multimap \phi_{2}\right)[1], \Delta . W$, and clearly the linear proof corresponding to this resource proof is $\Phi$.

$L^{\perp}$ : In this case, we have that $\Gamma=\phi^{\perp}, \Gamma^{\prime}$, and there is a proof of $\Gamma^{\prime} \vdash \phi, \Delta$ which is a subproof of $\Phi$. By the hypothesis, there are disjoint sets of variables $V$ and $W$ such that there is a resource proof of 
$\Gamma^{\prime} . V \vdash \phi[1], \Delta . W$, and hence there is a resource proof of $\Gamma^{\prime} . V,\left(\phi^{\perp}\right)[1] \vdash \Delta . W$, and clearly the linear proof corresponding to this resource proof is $\Phi$.

$R^{\perp}$ : In this case, we have that $\Delta=\phi^{\perp}, \Delta^{\prime}$, and there is a proof of $\Gamma, \phi \vdash \Delta^{\prime}$ which is a subproof of $\Phi$. By the hypothesis, there are disjoint sets of variables $V$ and $W$ such that there is a resource proof of $\Gamma . V, \phi[1] \vdash \Delta^{\prime} . W$, and hence there is a resource proof of $\Gamma . V \vdash\left(\phi^{\perp}\right)[1], \Delta^{\prime} . W$, and clearly the linear proof corresponding to this resource proof is $\Phi$.

The reader will have noted that in the application of any rule, it is always necessary for the expression for the principal formula of the rule to be 1 . In what follows we will omit an explicit statement of this requirement when it is obvious from the context.

\section{Linear additives and exponentials}

We now consider how to extend the approach of $\S 2$ to include additives and the exponentials of linear logic. As noted above, we only consider propositional fragments in this paper; the quantifiers per se provide no great technical problem (indeed, see [5] for a basic treatment).

Hence we now consider the class of formulæ defined in the following definition:

Definition 9 The formula of propositional linear logic PLL are as follows:

$$
p|\phi \otimes \phi| \phi \ngtr \phi|\phi \multimap \phi| \phi \& \phi|\phi \oplus \phi| ! \phi|? \phi| \phi^{\perp}|\mathbf{1}| \perp|\mathbf{0}| \top
$$

where $p$ is an atom.

It is straightforward to extend the rules of resource derivations to this class of formulæ, as below.

One point to note is that the Boolean expression technique discussed above may be used to characterize the choice of formula in the $\oplus \mathrm{R}$ and \& $\mathrm{L}$ rules. For example, the $\oplus \mathrm{R}$ rule requires a choice to be made between $\phi_{1}$ and $\phi_{2}$, as below.

$$
\oplus \mathrm{R} \frac{\Gamma \vdash \phi_{i}, \Delta}{\Gamma \vdash \phi_{1} \oplus \phi_{2}, \Delta}
$$

However, it is clear that by attaching a Boolean variable to $\phi_{1}$ and its complement to $\phi_{2}$ we are able to use the same constraint technique as above. This requires the rule to be reformulated as

$$
\oplus \mathrm{R} \frac{\Gamma \vdash \phi_{1}[x], \phi_{2}[\bar{x}], \Delta}{\Gamma \vdash\left(\phi_{1} \oplus \phi_{2}\right)[1], \Delta}
$$

Similar remarks apply to the \&L rule.

The exponentials are also straightforward, as they do not introduce any distribution problems. It should be noted, though, that the technique of attaching Boolean expressions to formulæ may be adapted to whatever approach is taken to the rules involving exponentials. For example, it is well-known that the weakening rules (W!L and W?R) may be permuted upwards, and hence incorporated into the leaf rules.

Also, the rules !L and C!L may be combined into one, as below [15]:

$$
\frac{\Gamma, \phi, ! \phi \vdash \Delta}{\Gamma, ! \phi \vdash \Delta}
$$

In either case, it is not hard to see how to adapt the resource versions of these rules to incorporate these adaptations. Hence here we consider only the "naive" exponential rules, as variations of them, as mentioned above, constitute only minor changes to the rules below. 
Definition 10 We define the following sequent calculus with constraints for PLL:

$$
\begin{aligned}
& \text { Axiom } \frac{e_{1}=e_{2}=1 \forall e_{3} \in \exp (\Gamma \cup \Delta) e_{3}=0}{\Gamma, p\left[e_{1}\right] \vdash p\left[e_{2}\right], \Delta} \\
& \perp \mathrm{L} \quad \frac{\forall e_{2} \in \exp (\Gamma \cup \Delta) e_{2}=0}{\Gamma, \perp\left[e_{1}\right] \vdash \Delta} e_{1}=1 \\
& \perp \mathrm{R} \quad \frac{\Gamma \vdash \Delta}{\Gamma \vdash \perp[e], \Delta} e=1 \\
& 1 \mathrm{~L} \quad \frac{\Gamma \vdash \Delta}{\Gamma, \mathbf{1}[e] \vdash \Delta} e=1 \\
& \text { 1R } \quad \frac{\forall e_{2} \in \exp (\Gamma \cup \Delta) e_{2}=0}{\Gamma \vdash \mathbf{1}\left[e_{1}\right], \Delta} e_{1}=1 \\
& \text { OL } \quad \frac{}{\Gamma, \mathbf{0}\left[e_{1}\right] \vdash \Delta} e_{1}=1 \\
& \mathrm{TR} \quad \frac{e_{1}=1}{\Gamma \vdash \top\left[e_{1}\right] \Delta} \\
& \begin{array}{ll}
\text { \&L } & \frac{\Gamma . V, \phi_{1}[e] \vdash \Delta . W \quad \Gamma \cdot \bar{V}, \phi_{2}}{\Gamma,\left(\phi_{1} \ngtr \phi_{2}\right)[e] \vdash} \\
\otimes \mathrm{L} & \frac{\Gamma, \phi_{1}[e], \phi_{2}[e] \vdash \Delta}{\Gamma,\left(\phi_{1} \otimes \phi_{2}\right)[e] \vdash \Delta} e=1
\end{array} \\
& \multimap \mathrm{L} \quad \frac{\Gamma . V \vdash \phi_{1}[e], \Delta . W \quad \Gamma \cdot \bar{V}, \phi_{2}[e] \vdash \Delta . \bar{W}}{\Gamma,\left(\phi_{1} \multimap \phi_{2}\right)[e] \vdash \Delta} e=1 \quad \multimap \mathrm{R} \quad \frac{\Gamma, \phi_{1}[e] \vdash \phi_{2}[e], \Delta}{\Gamma \vdash\left(\phi_{1} \multimap \phi_{2}\right)[e], \Delta} e=1 \\
& \mathrm{~L}^{\perp} \quad \frac{\Gamma \vdash \phi[e], \Delta}{\Gamma, \phi^{\perp}[e] \vdash \Delta} e=1 \\
& \mathrm{R}^{\perp} \quad \frac{\Gamma, \phi[e] \vdash \Delta}{\Gamma \vdash \phi^{\perp}[e], \Delta} e=1 \\
& \oplus \mathrm{L} \quad \frac{\Gamma, \phi_{1}[e] \vdash \Delta \quad \Gamma, \phi_{2}[e] \vdash \Delta}{\Gamma,\left(\phi_{1} \oplus \phi_{2}\right)[e] \vdash \Delta} e=1 \\
& \oplus \mathrm{R} \quad \frac{\Gamma \vdash \phi_{1}[x], \phi_{2}[\bar{x}], \Delta}{\Gamma \vdash\left(\phi_{1} \oplus \phi_{2}\right)[e], \Delta} e=1 \\
& \text { \&L } \quad \frac{\Gamma, \phi_{1}[x], \phi_{2}[\bar{x}] \vdash \Delta}{\Gamma,\left(\phi_{1} \& \phi_{2}\right)[e] \vdash \Delta} e=1 \\
& \text { \&R } \quad \frac{\Gamma \vdash \phi_{1}[e], \Delta \quad \Gamma \vdash \phi_{2}[e], \Delta}{\Gamma \vdash\left(\phi_{1} \& \phi_{2}\right)[e], \Delta} e=1 \\
& \text { !L } \quad \frac{\Gamma, \phi[e] \vdash \Delta}{\Gamma, ! \phi[e] \vdash \Delta} e=1 \\
& \text { !R } \quad \frac{! \Gamma \vdash \phi[e], ? \Delta}{! \Gamma \vdash ! \phi[e], ? \Delta} e=1 \\
& \text { ?L } \quad \frac{! \Gamma, \phi[e] \vdash ? \Delta}{! \Gamma, ? \phi[e] \vdash ? \Delta} e=1 \\
& \text { ?R } \quad \frac{\Gamma \vdash \phi[e], \Delta}{\Gamma \vdash ? \phi[e], \Delta} e=1 \\
& W ! \mathrm{L} \quad \frac{\Gamma \vdash \Delta}{\Gamma, ! \phi[e] \vdash \Delta} e=1 \\
& W ? \mathrm{R} \quad \frac{\Gamma \vdash \Delta}{\Gamma \vdash ? \phi[e], \Delta} e=1 \\
& C ! \mathrm{L} \quad \frac{\Gamma, ! \phi[e], ! \phi[e] \vdash \Delta}{\Gamma, ! \phi[e] \vdash \Delta} e=1 \\
& C ? \mathrm{R} \quad \frac{\Gamma \vdash ? \phi[e], ? \phi[e], \Delta}{\Gamma \vdash ? \phi[e], \Delta} e=1
\end{aligned}
$$

where the rules $\otimes R, 8 L$ and $\multimap L$ have the side-condition that $V$ and $W$ are disjoint sets of Boolean variables, none of which occur in $\Gamma, \Delta, \phi_{1}$ or $\phi_{2}$.

It is then straightforward to show the soundness of resource proofs for PLL.

Proposition 3 (soundness of resource proofs) Let $\Gamma \vdash \Delta$ be a resource sequent in PLL. If $\Gamma \vdash \Delta$ has a resource proof $R$ with Boolean assignment $I$, then the linear proof tree corresponding to $R$ is a linear proof of $\Gamma[I]^{1} \vdash \Delta[I]^{1}$.

Proof By induction on the structure of resource proofs. Assume that $\Gamma \vdash \Delta$ has a resource proof.

In the base case, the sequent is the conclusion of either the Axiom, $\perp \mathrm{L}, 1 \mathrm{R}, \mathrm{OL}$ or $T \mathrm{R}$ rules, and it is clear that the result holds in each of these cases.

Hence we assume that the result holds for all provable sequents whose resource proof is no more than a given size.

Consider the last rule used in the proof. We only give the argument for the cases $\otimes \mathrm{L}, \otimes R, \& \mathrm{~L}, \& \mathrm{R}, \mathrm{C} ! \mathrm{L}$; the others are similar. 
\&L: In this case, we have that $\Gamma=\left(\phi_{1} \& \phi_{2}\right)[1], \Gamma^{\prime}$, and there is a resource proof of $\Gamma^{\prime}, \phi_{1}[x], \phi_{2}[\bar{x}] \vdash \Delta$. By the hypothesis, there is a linear proof of $\Gamma^{\prime}[I]^{1},\left(\phi_{1}[x]\right)[I]^{1},\left(\phi_{2}[\bar{x}]\right)[I]^{1} \vdash \Delta[I]^{1}$ for some Boolean assignment $I$, which is clearly either $\Gamma^{\prime}[I]^{1}, \phi_{1} \vdash \Delta[I]^{1}$ or $\Gamma^{\prime}[I]^{1}, \phi_{2} \vdash \Delta[I]^{1}$, as required.

\&R: In this case, we have that $\Delta=\left(\phi_{1} \& \phi_{2}\right)[1], \Delta^{\prime}$, and $\Gamma \vdash \phi_{1}[1], \Delta^{\prime}$ and $\Gamma \vdash \phi_{2}[1], \Delta^{\prime}$ both have resource proofs. By the hypothesis, we have that there are linear proofs of $\Gamma[I]^{1} \vdash\left(\phi_{1}\right)[I]^{1}, \Delta^{\prime}[I]^{1}$ and $\Gamma[I]^{1} \vdash$ $\left(\phi_{2}\right)[I]^{1}, \Delta^{\prime}[I]^{1}$, and so there is a linear proof of $\Gamma[I]^{1} \vdash\left(\phi_{1} \& \phi_{2}\right)[I]^{1}, \Delta^{\prime}[I]^{1}$ which is just $\Gamma[I]^{1} \vdash \Delta[I]^{1}$, as required.

$\otimes \mathrm{L}$ : In this case, we have that $\Gamma=\left(\phi_{1} \otimes \phi_{2}\right)[1], \Gamma^{\prime}$, and there is a resource proof of $\Gamma^{\prime}, \phi_{1}, \phi_{2} \vdash \Delta$. By the hypothesis, there is a linear proof of $\Gamma^{\prime}[I]^{1}, \phi_{1}[I]^{1}, \phi_{2}[I]^{1} \vdash \Delta[I]^{1}$ for some Boolean assignment $I$, and hence there is a linear proof of $\Gamma^{\prime}[I]^{1}\left(\phi_{1} \otimes \phi_{2}\right)[I]^{1} \vdash \Delta[I]^{1}$, which is just $\Gamma^{\prime}[I]^{1} \vdash \Delta[I]^{1}$, as required.

$\otimes \mathrm{R}$ : In this case, we have that $\Delta=\left(\phi_{1} \otimes \phi_{2}\right)[1], \Delta^{\prime}$, and $\Gamma . V \vdash \phi_{1}[1], \Delta^{\prime} . W$ and $\Gamma \cdot \bar{V} \vdash \phi_{2}[1], \Delta^{\prime} \cdot \bar{W}$ both have resource proofs for some disjoint sets of Boolean variables $V$ and $W$. By the hypothesis, we have that there are linear proofs of $(\Gamma . V)[I]^{1} \vdash\left(\phi_{1}\right)[I]^{1},\left(\Delta^{\prime} . W\right)[I]^{1}$ and $(\Gamma \cdot \bar{V})[I]^{1} \vdash\left(\phi_{2}\right)[I]^{1},\left(\Delta^{\prime} . \bar{W}\right)[I]^{1}$ (recall that $I$ must be an assignment of all Boolean variables in the proof), and so there is a linear proof of $(\Gamma . V)[I]^{1},(\Gamma \cdot \bar{V})[I]^{1} \vdash\left(\phi_{1} \otimes \phi_{2}\right)[I]^{1},\left(\Delta^{\prime} . W\right)[I]^{1},\left(\Delta^{\prime} . \bar{W}\right)[I]^{1}$ which is just $\Gamma[I]^{1} \vdash \Delta[I]^{1}$, as required.

C!L: In this case, we have that $\Gamma=! \phi[1], \Gamma^{\prime}$, and there is a resource proof of $\Gamma^{\prime}, ! \phi[1], ! \phi[1] \vdash \Delta$. By the hypothesis, there is a linear proof of $\Gamma^{\prime}[I]^{1}, ! \phi[I]^{1}, ! \phi[I]^{1} \vdash \Delta[I]^{1}$ for some Boolean assignment $I$, and hence there is a linear proof of $\Gamma^{\prime}[I]^{1}, ! \phi[I]^{1} \vdash \Delta[I]^{1}$, which is just $\Gamma[I]^{1} \vdash \Delta[I]^{1}$, as required.

As previously, we require the following lemma in order to show the completeness of resource proofs for PLL.

Lemma 2 Let $\Gamma \vdash \Delta$ be a resource sequent in PLL. If $\Gamma \vdash \Delta$ has a closed resource derivation $R$, then $\Gamma, \phi[0] \vdash \Delta$ and $\Gamma \vdash \phi[0], \Delta$ also have closed resource derivations $R_{1}$ and $R_{2}$ respectively. Moreover, for any total assignment $I$ of the Boolean variables in $R$, the linear proof tree corresponding to $R$ under $I$ is the same as the linear proof trees corresponding to $R_{1}$ and $R_{2}$ respectively under $I$.

Proof The proof is similar to that of Lemma 1, and hence is omitted.

We are now in a position to show the completeness of resource proofs for PLL.

Proposition 4 (completeness of resource proofs) Let $\Gamma \vdash \Delta$ be a sequent in PLL. If $\Gamma \vdash \Delta$ has a proof $\Phi$ in PLL, then there are disjoint sets of Boolean variables $V$ and $W$ such that $\Gamma . V \vdash \Delta . W$ has a resource proof $R$ and the linear proof tree corresponding to $R$ is $\Phi$.

Proof By induction on the structure of resource proofs. Assume that $\Gamma \vdash \Delta$ has a resource proof.

In the base case, the sequent is the conclusion of either the Axiom, $\perp \mathrm{L}, 1 \mathrm{R}, \mathrm{OL}$ or $T \mathrm{R}$ rules, and it is clear that the result holds in each of these cases.

Hence we assume that the result holds for all provable sequents whose resource proof is no more than a given size.

Consider the last rule used in the proof. We only give the argument for the cases $\otimes \mathrm{L}, \otimes R, \& L, \& R, C ! L$; the others are similar.

$\otimes \mathrm{L}$ : In this case, we have that $\Gamma=\phi_{1} \otimes \phi_{2}, \Gamma^{\prime}$, and there is a proof of $\Gamma^{\prime}, \phi_{1}, \phi_{2} \vdash \Delta$ which is a subproof of $\Phi$. By the hypothesis, there are disjoint sets of variables $V$ and $W$ such that there is a resource proof of $\Gamma^{\prime} . V, \phi_{1}[1], \phi_{2}[1] \vdash \Delta . W$, and hence there is a resource proof of $\Gamma^{\prime} . V,\left(\phi_{1} \otimes \phi_{2}\right)[1] \vdash \Delta . W$, and clearly the linear proof corresponding to this resource proof is $\Phi$.

$\otimes \mathrm{R}$ : In this case, we have that $\Gamma=\Gamma_{1}, \Gamma_{2}, \Delta=\phi_{1} \otimes \phi_{2}, \Delta_{1}, \Delta_{2}$ such that $\Gamma_{1} \vdash \phi_{1}, \Delta_{1}$ and $\Gamma_{2} \vdash \phi_{2}, \Delta_{2}$ both have proofs in the linear sequent calculus which are subproofs of $\Phi$. Hence by the hypothesis there are disjoint sets of Boolean variables $V_{1}, V_{2}, W_{1}, W_{2}$ such that $\Gamma_{1} . V_{1} \vdash \phi_{1}[1], \Delta_{1} . W_{1}$ and $\Gamma_{2} . V_{2} \vdash \phi_{2}[1], \Delta_{2} . W_{2}$ have resource proofs, (and moreover the linear proofs corresponding to each resource proof is the appropriate subproof of $\Phi$ ) and so by Lemma 2 , there are closed resource derivations of $\Gamma_{1} \cdot V_{1}, \Gamma_{2} \cdot\{0\}^{n} \vdash$ 
$\phi_{1}[1], \Delta_{1} \cdot W_{1}, \Delta_{2} .\{0\}^{n}$ and $\Gamma_{1} \cdot\{0\}^{n}, \Gamma_{2} . V_{2} \vdash \phi_{2}[1], \Delta_{1} \cdot\{0\}^{n}, \Delta_{2} . W_{2}$. Hence there are new disjoint sets of Boolean variables (i.e., not occurring anywhere in the above two resource sequents) $V$ and $W$ and a total assignment $I$ of $V \cup W$ such that $\left(\Gamma_{1} \cdot V_{1}, \Gamma_{2} \cdot V_{2}\right) . V \vdash \phi_{1}[1],\left(\Delta_{1} \cdot W_{1}, \Delta_{2} \cdot W_{2}\right) . W$ and $\left(\Gamma_{1} \cdot V_{1}, \Gamma_{2} \cdot V_{2}\right) \cdot \bar{V} \vdash$ $\phi_{2}[1],\left(\Delta_{1} . W_{1}, \Delta_{2} \cdot W_{2}\right) \cdot \bar{W}$ have resource proofs, and so there is a resource proof of $\Gamma_{1} \cdot V_{1}, \Gamma_{2} . V_{2} \vdash\left(\phi_{1} \otimes\right.$ $\left.\phi_{2}\right)[1], \Delta_{1} . W_{1}, \Delta_{2} . W_{2}$, i.e., $\Gamma . V^{\prime} \vdash\left(\phi_{1} \otimes \phi_{2}\right)[1], \Delta . W^{\prime}$ for some disjoint sets of Boolean variables $V^{\prime}$ and $W^{\prime}$, and clearly the linear proof corresponding to this resource proof is $\Phi$.

\& L: In this case, we have that $\Gamma=\phi_{1} \& \phi_{2}, \Gamma^{\prime}$, and there is a proof of $\Gamma^{\prime}, \phi_{i} \vdash \Delta$ which is a subproof of $\Phi$. Without loss of generality, let $i=1$. By the hypothesis, there are disjoint sets of variables $V$ and $W$ such that there is a resource proof of $\Gamma^{\prime} . V, \phi_{1}[1] \vdash \Delta . W$, and by Lemma 2 there is a resource proof of $\Gamma^{\prime} . V, \phi_{1}[1], \phi_{2}[0] \vdash \Delta . W$, i.e., there is a resource proof of $\Gamma^{\prime} . V, \phi_{1}[x], \phi_{2}[\bar{x}] \vdash \Delta . W$, giving us a resource proof of $\Gamma^{\prime} . V,\left(\phi_{1} \& \phi_{2}\right)[1] \vdash \Delta . W$, and clearly the linear proof corresponding to this resource proof is $\Phi$.

\& R: In this case, we have that $\Delta=\phi_{1} \& \phi_{2}, \Delta^{\prime}$ such that $\Gamma \vdash \phi_{1}, \Delta^{\prime}$ and $\Gamma \vdash \phi_{2}, \Delta$ both have proofs in the linear sequent calculus which are subproofs of $\Phi$. Hence by the hypothesis there are disjoint sets of Boolean variables $V_{1}, V_{2}, W_{1}, W_{2}$ such that $\Gamma . V_{1} \vdash \phi_{1}[1], \Delta . W_{1}$ and $\Gamma . V_{2} \vdash \phi_{2}[1], \Delta . W_{2}$ have resource proofs, (and moreover the linear proofs corresponding to each resource proof is the appropriate subproof of $\Phi)$. Now as each of these has the property that the Boolean expression attached to each formula in the conclusion evaluates to 1 , we can choose $V_{1}=V_{2}$ and $W_{1}=W_{2}$, and hence we have a resource proof of $\Gamma . V_{1} \vdash\left(\phi_{1} \& \phi_{2}\right)[1], \Delta . W_{1}$, and clearly the linear proof corresponding to this resource proof is $\Phi$.

C!L: In this case, we have that $\Gamma=! \phi, \Gamma^{\prime}$, and there is a proof of $\Gamma^{\prime}, ! \phi, ! \phi \vdash \Delta$ which is a subproof of $\Phi$. By the hypothesis, there are disjoint sets of variables $V$ and $W$ such that there is a resource proof of $\Gamma^{\prime} . V, ! \phi[1], ! \phi[1] \vdash \Delta . W$, and hence there is a resource proof of $\Gamma^{\prime} . V, ! \phi[1] \vdash \Delta . W$, and clearly the linear proof corresponding to this resource proof is $\Phi$.

It should be noted that the reason that it is straightforward to adapt the $\oplus \mathrm{R}$ and \& $\mathrm{L}$ rules as above is that the Boolean constraints to be solved are of the same form as those in $\S 2$, i.e., determining the satisfiability of expressions such as $x . y . \bar{z}$. This may be thought of as determining the truth of the existentially quantified expression $\exists x \cdot \exists y \cdot \exists z \cdot(x \cdot y \cdot \bar{z})$.

Now if we were to consider universally quantified expressions as well, then we arrive at a technique similar to the "slices" used in proof nets for additive rules [3]. In particular, we could re-write the $\otimes \mathrm{R}$ rule as

$$
\frac{\Gamma \vdash \phi_{1}[x], \phi_{2}[\bar{x}], \Delta}{\Gamma \vdash \phi_{1} \otimes \phi_{2}, \Delta} \otimes \mathrm{R}
$$

which would result in a universally quantified expression. Hence the complexity of finding solutions is increased, but for the benefit of providing an algebraic interpretation of proofs in which the additives are taken as basic, and the multiplicatives introduced via the Boolean constraints. This contrasts with proof-nets, in which the additives are introduced to a multiplicative system by means of the Boolean expressions. Further development on this point is beyond the scope of this paper; we will take up this thread in a subsequent paper.

\section{Bunched additives}

We now turn to a different extension of MLL, in which the additives are handle rather differently. Roughly, in the logic of bunched implications, BI, there are two implications - one multiplicative, and one additive. This necessitates a more complex structure in the antecedent, as we need to be able to distinguish additive contexts from multiplicative contexts. However, as we shall see, the same basic technique as above may be applied to the problem of distribution of formulæ across multiplicative branches of a proof.

In BI, there are multiplicative versions of conjunction and disjunction (as in linear logic), but also of implication (unlike linear logic). Hence we have the following two rules for implications in succedents:

$$
\rightarrow \mathrm{R} \frac{\Gamma ; \phi_{1} \vdash \phi_{2}}{\Gamma \vdash \phi_{1} \rightarrow \phi_{2}} \quad * \mathrm{R} \frac{\Gamma, \phi_{1} \vdash \phi_{2}}{\Gamma \vdash \phi_{1} * \phi_{2}}
$$

These rules thus show that implication is intimately associated with the way in which antecedents are formed. In particular, because of the existence of the two implication rules above, it is necessary to have two constructors for 


$\Gamma::=\quad \begin{array}{lr}\phi & \text { propositional assumption } \\ \emptyset_{m} & \text { multiplicative unit } \\ \Gamma, \Gamma & \text { multiplicative combination } \\ \emptyset_{a} & \text { additive unit } \\ \Gamma ; \Gamma & \text { additive combination }\end{array}$

Figure 1: Bunches

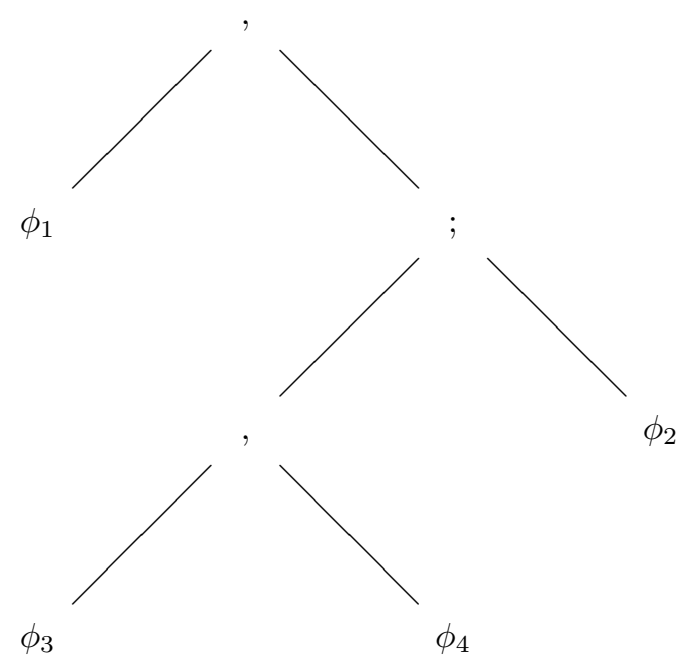

Figure 2: An example bunch

antecedents: one multiplicative, written as “," and logically equivalent to BI's multiplicative conjunction, $*$, and one additive, written as ";" and logically equivalent to BI's additive conjunction, $\wedge .{ }^{\beta}$

Thus antedecents in BI are not simply finite sequences, as in linear logic, but finite trees, with the leaf nodes being formulæ, and the internal nodes denoted by either “,” or “;”, and are referred to as bunches. The grammar of bunches is given in Figure 1 .

For example, the bunch $\phi_{1},\left(\left(\phi_{3}, \phi_{4}\right) ; \phi_{2}\right)$ may be drawn as in Figure2.

The main point of the definition of bunches is that "“; admits the structural properties of Weakening and Contraction, whereas "," does not: this distinction allows the correct formulation of the two implications. Bunches may be represented using lists of lists, etc., as described in [12]. We write $\Gamma(\Delta)$, and refer to $\Delta$ as a sub-bunch of $\Gamma$, for a bunch $\Gamma$ in which $\Delta$ appears as a sub-tree and write $\Gamma\left[\Delta^{\prime} / \Delta\right]$ for $\Gamma$ with $\Delta$ replaced by $\Delta^{\prime}$. We write $\Gamma(-)$ to denote a bunch $\Gamma$ which is incomplete and which may be completed by placing a bunch in its hole, and will use this notation to refer to that part of $\Gamma(\Delta)$ which is not part of $\Delta$. We require that "," and "," be commutative monoids, giving rise to the coherent equivalence, $\Gamma \equiv \Gamma^{\prime}$, as follows:[]

CoHerent Equivalence: $\Gamma \equiv \Gamma^{\prime}$

1 Commutative monoid equations for $\emptyset_{a}$ and “;”.

\footnotetext{
${ }^{3}$ The underlying semantic strycture of BI's proofs, which can be represented as the terms of a simply-typed $\lambda$-calculus, is given by a bi-cartesian doubly closed category $18,10,11$, which carries two closed structures, one symmetric monoidal and one bi-cartesian. There are many examples of such categories $\$ 8,10,11]$.

${ }^{4}$ A multiple-conclusioned version of BI, with disjunctive bunched structure in succedents, may also be formulated [11]. For simplcity, we restrict our attention here to the single-conclusioned case.

${ }^{5}$ The multiplicative operation, ",", need not, in general, be commutative and, indeed, such non-commutative versions of BI are possible [11].
} 
2 Commutative monoid equations for $\emptyset_{m}$ and ",".

3 Congruence: if $\Delta \equiv \Delta^{\prime}$ then $\Gamma(\Delta) \equiv \Gamma\left(\Delta^{\prime}\right)$.

Note that "," and "," do not distribute over one another. We use = for syntactic identity of bunches.

BI may also be understood semantically [8, 10, 11]. The key structure is that of a preordered monoid, $\mathcal{M}=$ $(M, \cdot, e, \sqsubseteq)$ which provides the worlds for a Kripke-style forcing semantics. The additives receive a semantics in the usual intuitionistic way, whereas the multiplicatives receive a semantics in the style of Kripke, Urquhart and Pym [7, 16, 8, 10, 11]:

$$
\begin{gathered}
m \models \phi * \psi \quad \text { iff } \quad \text { for all } n \text { such that } n \models \phi, m \cdot n \models \psi \\
m \models \phi * \psi \quad \text { iff } \quad \text { there exist } n \text { and } n^{\prime}, \text { where } m \sqsubseteq n \cdot n^{\prime} \text {, such that } n \models \phi \text { and } n^{\prime} \models \psi .
\end{gathered}
$$

Categorically, such a semantics can be formulated in doubly closed categories of the form $\operatorname{Set}^{C}$, where $C$ is a small symmetric monoidal [8, 10, 11]. This semantics may be interpreted as being a model of the notion of resource: it may be argued that the following are a basic set of assumptions about resources:

- There should be a zero resource; call it $e$;

- The should be a combination of resources; call it ·;

- The should be a a comparison of resources; call it $\sqsubseteq$.

The assumptions lead to the forcing semantics based on preordered monoids. The leading characteristic of this resource semantics is the sharing interpretation: we can analyse the sharing and privacy of the resources accessed by co-existing computations. The topics, including soundness and completeness theorems and computationally natural concrete models, are discussed fully in [8, 10, 11, 9]. The logical view of resources sketched here should be contrasted with that which is available for linear logic, in which a proposition is interpreted directly as a resource and its number of uses is counted.

For further information on $\mathbf{B I}$, the reader is referred to [8, 10, 11]. A good summary of the use of bunched structure in substructural logic may be found in [13, 12].

Below is (a version of) the sequent calculus LBI for propositional BI [8, 10, 11].

Definition 11 The calculus LBI may be defined as follows: 


$$
\begin{aligned}
& \text { Axiom } \frac{}{\phi \vdash \phi} \quad \text { E } \quad \frac{\Gamma \vdash \phi}{\Delta \vdash \phi} \text { where } \Gamma \equiv \Delta \\
& \mathrm{W} \quad \frac{\Gamma(\Delta) \vdash \phi}{\Gamma\left(\Delta ; \Delta^{\prime}\right) \vdash \phi} \quad \mathrm{C} \quad \frac{\Gamma(\Delta ; \Delta) \vdash \phi}{\Gamma(\Delta) \vdash \phi} \\
& I \mathrm{~L} \quad \frac{\Gamma\left(\emptyset_{m}\right) \vdash \phi}{\Gamma(I) \vdash \phi} \quad I \mathrm{R} \quad \frac{\emptyset_{m} \vdash I}{} \\
& 1 \mathrm{~L} \quad \frac{\Gamma\left(\emptyset_{a}\right) \vdash \phi}{\Gamma(1) \vdash \phi} \quad \text { 1R } \frac{\bar{\emptyset}+1}{\emptyset_{a} \vdash 1} \\
& \perp \mathrm{L} \quad \overline{\perp \vdash \phi} \\
& * \mathrm{~L} \quad \frac{\Gamma \vdash \phi_{1} \quad \Delta\left(\Delta^{\prime}, \phi_{2}\right) \vdash \phi}{\Delta\left(\Delta^{\prime}, \Gamma, \phi_{1} * \phi_{2}\right) \vdash \phi} \quad * \mathrm{R} \quad \frac{\Gamma, \phi_{1} \vdash \phi_{2}}{\Gamma \vdash \phi_{1} * \phi_{2}} \\
& * \mathrm{~L} \quad \frac{\Gamma\left(\phi_{1}, \phi_{2}\right) \vdash \phi}{\Gamma\left(\phi_{1} * \phi_{2}\right) \vdash \phi} \quad * \mathrm{R} \quad \frac{\Gamma \vdash \phi_{1} \Delta \vdash \phi_{2}}{\Gamma, \Delta \vdash \phi_{1} * \phi_{2}} \\
& \rightarrow \mathrm{L} \quad \frac{\Gamma \vdash \phi_{1} \Delta\left(\Gamma ; \phi_{2}\right) \vdash \phi}{\Delta\left(\Gamma ; \phi_{1} \rightarrow \phi_{2}\right) \vdash \phi} \rightarrow \mathrm{R} \quad \frac{\Gamma ; \phi_{1} \vdash \phi_{2}}{\Gamma \vdash \phi_{1} \rightarrow \phi_{2}} \\
& \wedge \mathrm{L} \quad \frac{\Gamma\left(\phi_{1} ; \phi_{2}\right) \vdash \phi}{\Gamma\left(\phi_{1} \wedge \phi_{2}\right) \vdash \phi} \quad \wedge \mathrm{R} \quad \frac{\Gamma \vdash \phi_{1} \Gamma \vdash \phi_{2}}{\Gamma \vdash \phi_{1} \wedge \phi_{2}} \\
& \vee \mathrm{L} \quad \frac{\Gamma\left(\phi_{1}\right) \vdash \phi \quad \Gamma\left(\phi_{2}\right) \vdash \phi}{\Gamma\left(\phi_{1} \vee \phi_{2}\right) \vdash \phi} \quad \vee \mathrm{R} \quad \frac{\Gamma \vdash \phi_{i}}{\Gamma \vdash \phi_{1} \vee \phi_{2}}
\end{aligned}
$$

We will refer to the Axiom, $I R, 1 R$ and $\perp L$ rules as leaf rules.

Note that, since LBI admits Cut-elimination [11], we omit the Cut rule. Also, we have used the additive version of the rules $\wedge \mathrm{R}, \rightarrow \mathrm{L}$ and $\vee \mathrm{L}$ rules (see [8, 10, 11] for details). This is done because the derivability of these rules in LBI may be interpreted as showing that these rules need not introduce any new distribution problems. Note also that, as in [8, 10, 11], we could have used a similarly additive form of the Axiom rule, viz.:

$$
\text { Axiom } \overline{\phi ; \Gamma \vdash \phi}
$$

However, we have chosen not to do this as it unnecessarily complicates the structure of the antecedent. Recall that the basic technique is to relax the multiplicative rules so that "extra" formulæ are present in the sequent, and then the constraints are used to determine the distribution of all formulæ across multiplicative branches. In the linear case, as antecedents are multiplicative, there is no issue. In the BI case, the antecedent could be additive, as in the above rule. The resource version of the Axiom rule would then be

$$
\operatorname{Axiom} \frac{\forall e \in \exp (\Delta) e=0}{\left(\phi\left[e_{1}\right] ; \Gamma\right), \Delta \vdash \phi} e_{1}=1,
$$

which seems unnecessarily complex, especially as the rules of weakening and contraction are still required. Hence we use the original form of the Axiom rule but the additive versions of the $\wedge \mathrm{R}, \rightarrow \mathrm{L}$ and $\vee \mathrm{L}$ rules.

Now the only rules which require attention are the leaf rules leaves of the tree (Axiom, $I \mathrm{R}, \perp \mathrm{L}, 1 \mathrm{R})$ and $* \mathrm{~L}($ as $* \mathrm{R}$ is precisely the same rule as for linear logic). We reformulate the leaf rules as follows: 


$$
\begin{array}{lc}
\text { Axiom } \frac{\forall e \in \exp (\Delta) e=0}{\phi\left[e_{1}\right], \Delta \vdash \phi} e_{1}=1 & \perp \mathrm{L} \frac{\forall e \in \exp (\Delta) e=0}{\perp\left[e_{1}\right], \Delta \vdash \phi} e_{1}=1 \\
\operatorname{IR} \frac{\forall e \in \exp (\Delta) e=0}{\emptyset_{m}\left[e_{1}\right], \Delta \vdash I} e_{1}=1 & 1 \mathrm{R} \frac{\forall e \in \exp (\Delta) e=0}{\emptyset_{a}\left[e_{1}\right], \Delta \vdash 1} e_{1}=1
\end{array}
$$

Note that as LBI is single-conclusioned, there is no need to consider distribution on the right of $\vdash$, and hence the formulæ in the succedents never require a Boolean expression. Otherwise, these are little different from similar rules in linear logic.

The only remaining rule is $* \mathrm{~L}$. Here the main point to note is that we need to take account of the bunch structure, but otherwise this is handled similarly to the $-\mathrm{L}$ rule in linear logic. The new form of the rule is then

$$
\frac{\Gamma . V \vdash \phi_{1} \quad \Delta\left(\Gamma \cdot \bar{V}, \phi_{2}[e]\right) \vdash \phi}{\Delta\left(\Gamma,\left(\phi_{1} * \phi_{2}\right)[e]\right) \vdash \phi} e=1
$$

where $\Gamma, \phi_{1} * \phi_{2}$ is the maximal multiplicative super-bunch of $\phi_{1} * \phi_{2}$ in $\Delta\left(\Gamma, \phi_{1} * \phi_{2}\right)$. For example, given the sequent

$$
p,(q ;(r, s, t * u)) \vdash v
$$

we get the following inference step:

$$
\frac{r\left[x_{1}\right], s\left[x_{2}\right] \vdash t \quad p,\left(q ;\left(r\left[\overline{x_{1}}\right], s\left[\overline{x_{2}}\right], u[1]\right)\right) \vdash v}{p,(q ;(r, s,(t * u)[1])) \vdash v}
$$

Here $\Gamma=\{r, s\}$ and not $\{r\}$ or $\{s\}$. We formalize this notion below.

Definition 12 Let $\Delta(\Gamma)$ be a bunch. Then the maximal multiplicative super-bunch of $\Gamma$ in $\Delta$ is defined as follows:

- If $\Delta(\Gamma)=\Gamma$ or the parent of $\Gamma$ in $\Delta$ is “;”, then the maximal multiplicative super-bunch of $\Gamma$ in $\Delta$ is just $\Gamma$.

- If the parent of $\Gamma$ in $\Delta$ is “,", then the maximal multiplicative super-bunch of $\Gamma$ in $\Delta$ is the maximal multiplicative super-bunch of the parent of $\Gamma$ in $\Delta$.

Note that in this case we have to attach expressions to bunches. For example, consider the provable sequent $r,(s ; t) \vdash r * s$. A resource derivation of this sequent would be

$$
\frac{r[x],(s ; t)[y] \vdash r \quad \frac{r[\bar{x}], s[\bar{y}] \vdash s}{r[\bar{x}],(s ; t)[\bar{y}] \vdash s}}{r,(s ; t) \vdash r * s} \mathrm{~W}
$$

from which we get $x=1, y=0$, corresponding to the sequent proof below.

$$
\frac{r \vdash r \quad \frac{s \vdash s}{s ; t \vdash s}}{r,(s ; t) \vdash r * s} * \mathrm{R}
$$

Hence we need to attach expressions to bunches. However, it will not necessarily be correct to simply attach the expression to every formula in the bunch, as a multiplicative bunch may contain an additive sub-bunch. For example, in the sequent above, we wish to distribute the two sub-bunches $r$ and $(s ; t)$ across the two multiplicative branches, and not the three sub-bunches $r, s$ and $t$.

Hence we need to define the operation $\Gamma . V$ where $\Gamma$ is a bunch so that it respects this multiplicative structure.

Definition 13 Let $\Gamma$ be a bunch and $V$ a set of distinct variables. We define the operation $\Gamma . V$ as follows:

- If $\Gamma=\phi$, then $|V|=1$ and $\Gamma . V=\phi[x]$ 
- If $\Gamma=\left(\Gamma^{\prime} ; \Delta\right)$, then $|V|=1$ and $\Gamma . V=\left(\Gamma^{\prime} ; \Delta\right)[x]$

- If $\Gamma=\left(\Gamma^{\prime}, \Delta\right)$, then $\Gamma . V=\left(\Gamma^{\prime} . V_{1}, \Delta . V_{2}\right)$ where $V_{1}, V_{2}$ are disjoint sets of variables such that $V=V_{1} \cup V_{2}$.

Thus we have that $r,(s ; t) .\{x, y\}=r[x],(s ; t)[y]$.

Note that the linear version of this operation may be thought of as a bunch in which the only constructor is "“".

Note also that in the additive implication it is also useful to identify the maximal additive super-bunch of the principal formula. Doing so allows us to show that there is indeed no distribution problem arising from the additive form of the $\rightarrow \mathrm{L}$ rule, as then $\Gamma$ and $\Delta$ may easily be determined from the rule application.

For example, given the sequent

$$
q,\left(p_{3} ; p_{1} ; p_{1} \rightarrow p_{2}\right) \vdash q * p_{2}
$$

it would seem appropriate to apply the $\rightarrow \mathrm{L}$ rule as follows

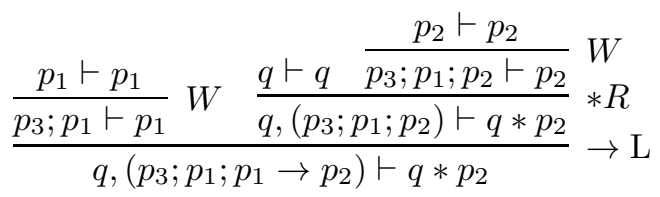

rather than

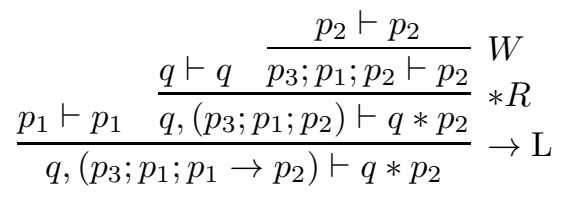

The former application avoids having to determine where $p_{3}$ will be used by sending it to each branch and deleting it (via the $\mathrm{W}$ rule) as required. However, we do not pursue this direction in this paper, as it has no effect on the issues of distribution.

We get the following rules:

Definition 14 We define the following sequent calculus with constraints for BI: 


$$
\begin{aligned}
& \text { Axiom } \quad \frac{\forall e \in \exp (\Delta) e=0}{\phi\left[e_{1}\right], \Delta \vdash \phi} e_{1}=1 \quad \text { E } \quad \frac{\Gamma[e] \vdash \phi}{\Delta[e] \vdash \phi} e=1 \text { where } \Gamma \equiv \Delta \\
& \mathrm{W} \quad \frac{\Gamma(\Delta[e]) \vdash \phi}{\Gamma\left(\left(\Delta ; \Delta^{\prime}\right)[e]\right) \vdash \phi} e=1 \quad \mathrm{C} \quad \frac{\Gamma(\Delta[e] ; \Delta[e]) \vdash \phi}{\Gamma(\Delta[e]) \vdash \phi} e=1 \\
& I \mathrm{~L} \quad \frac{\Gamma\left(\emptyset_{m}[e]\right) \vdash \phi}{\Gamma(I[e]) \vdash \phi} e=1 \quad I \mathrm{R} \quad \frac{\forall e \in \exp (\Delta) e=0}{\emptyset_{m}\left[e_{1}\right], \Delta \vdash I} e_{1}=1 \\
& 1 \mathrm{~L} \quad \frac{\Gamma\left(\emptyset_{a}[e]\right) \vdash \phi}{\Gamma(1[e]) \vdash \phi} e=1 \quad 1 \mathrm{R} \quad \frac{\forall e \in \exp (\Delta) e=0}{\emptyset_{a}\left[e_{1}\right], \Delta \vdash 1} e_{1}=1 \\
& \perp \mathbf{L} \quad \frac{\forall e \in \exp (\Delta) e=0}{\perp\left[e_{1}\right], \Delta \vdash \phi} e_{1}=1 \\
& * \mathrm{~L} \quad \frac{\left.\Gamma \cdot V \vdash \phi_{1} \quad \Delta\left(\Gamma \cdot \bar{V}, \phi_{2}[e]\right) \vdash \phi\right)}{\Delta\left(\Gamma, \phi_{1} * \phi_{2}[e]\right) \vdash \phi} e=1 \quad * \mathrm{R} \quad \frac{\Gamma, \phi_{1}[e] \vdash \phi_{2}}{\Gamma \vdash \phi_{1} * \phi_{2}} e=1 \\
& * \mathrm{~L} \quad \frac{\Gamma\left(\phi_{1}[e], \phi_{2}[e]\right) \vdash \phi}{\Gamma\left(\phi_{1} * \phi_{2}[e]\right) \vdash \phi} e=1 \quad * \mathrm{R} \quad \frac{\Gamma . V \vdash \phi_{1} \quad \Gamma . \bar{V} \vdash \phi_{2}}{\Gamma \vdash \phi_{1} * \phi_{2}} \\
& \rightarrow \mathrm{L} \quad \frac{\Gamma \vdash \phi_{1} \quad \Delta\left(\Gamma ; \phi_{2}[e]\right) \vdash \phi}{\Delta\left(\Gamma ; \phi_{1} \rightarrow \phi_{2}[e]\right) \vdash \phi} e=1 \quad \rightarrow \mathrm{R} \quad \frac{\Gamma ; \phi_{1}[e] \vdash \phi_{2}}{\Gamma \vdash \phi_{1} \rightarrow \phi_{2}} e=1 \\
& \wedge \mathrm{L} \quad \frac{\Gamma\left(\phi_{1}[e] ; \phi_{2}[e]\right) \vdash \phi}{\Gamma\left(\phi_{1} \wedge \phi_{2}[e]\right) \vdash \phi} e=1 \quad \wedge \mathrm{R} \quad \frac{\Gamma \vdash \phi_{1} \quad \Gamma \vdash \phi_{2}}{\Gamma \vdash \phi_{1} \wedge \phi_{2}} \\
& \vee \mathrm{L} \quad \frac{\Gamma\left(\phi_{1}[e]\right) \vdash \phi \quad \Gamma\left(\phi_{2}[e]\right) \vdash \phi}{\Gamma\left(\phi_{1} \vee \phi_{2}[e]\right) \vdash \phi} e=1 \quad \vee \mathrm{R} \quad \frac{\Gamma \vdash \phi_{i}}{\Gamma \vdash \phi_{1} \vee \phi_{2}}
\end{aligned}
$$

where the $* \mathrm{~L}$ rule has the side condition that $\Gamma, \phi_{1} * \phi_{2}$ is the maximal multiplicative super-bunch containing $\phi_{1} * \phi_{2}$, and the rules $* \mathrm{~L}$ and $* \mathrm{R}$ have the side condition that $V$ is a set of Boolean variables not occurring in $\Gamma$, $\Delta, \phi_{1}, \phi_{2}$ or $\phi$.

We are now in a position to define resource derivations for propositional BI. This is actually the same definition as Definition 7 .

Definition 15 A resource derivation is a tree regulated by the rules of the resource calculus in which each formula of the endsequent is assigned a distinct Boolean variable, together with a (possibly partial) assignment of the Boolean variables appearing in the derivation.

A resource derivation is total if its assignment of the Boolean variables is total. Otherwise, the resource derivation is partial. A resource derivation is closed if all of the leaves of the proof tree are leaf rules. A resource proof is a total, closed resource derivation in which all the Boolean variables in the endsequent are assigned the value 1.

As above, it is then straightforward to recover an LBI proof from a resource proof.

Definition 16 Let $R$ be a total resource derivation, with proof tree $T$ and Boolean assignment $I$. The LBI proof tree corresponding to $\mathrm{R}$ is the proof tree obtained by deleting from $T$ all formula whose Boolean expression evaluates to 0 under $I$.

For example, consider the resource derivation below.

$$
\frac{\frac{r\left[x_{1}\right], p\left[x_{2}\right] \vdash p}{r\left[x_{1}\right],(p ; t)\left[x_{2}\right] \vdash p} W \quad \frac{r\left[\overline{x_{1}} \cdot y_{1}\right],(p ; t)\left[\overline{x_{2}} \cdot y_{2}\right], q\left[y_{3}\right] \vdash q \quad r\left[\overline{x_{1}} \cdot \overline{y_{1}}\right],(p ; t)\left[\overline{x_{2}} \cdot \overline{y_{2}}\right], q\left[\overline{y_{3}}\right] \vdash r}{\frac{r\left[\overline{x_{1}}\right],(p ; t)\left[\overline{\bar{x}_{2}}\right], q \vdash q * r}{\left(r\left[\overline{x_{1}}\right],(p ; t)\left[\overline{x_{2}}\right], q\right) ; s \vdash q * r}} * \mathrm{R}}{(r,(p ; t), p * q[1]) ; s \vdash q * r} * \mathrm{~L}
$$


Solving the equations gives us

$$
x_{1}=0, x_{2}=1, y_{1}=0, y_{3}=1
$$

which in turn gives us the resource proof:

$$
\frac{\frac{r[0], p[1] \vdash p}{r[0],(p ; t)[1] \vdash p} W \quad \frac{r[0],(p ; t)[0], q[1] \vdash q \quad r[1],(p ; t)[0], q[0] \vdash r}{r[1],(p ; t)[0], q \vdash q * r}}{(r,(p ; t), p * q[1]) ; s \vdash q * r} W \mathrm{R}
$$

The corresponding LBI proof is then:

$$
\begin{gathered}
\frac{p \vdash p}{(p ; t) \vdash p} W \frac{\frac{q \vdash q \quad r \vdash r}{r, q \vdash q * r}}{\frac{(r, q) ; s \vdash q * r}{(r,(p ; t), p * q) ; s \vdash q * r}} W \\
* \mathrm{~L}
\end{gathered}
$$

Note that this proof could be part of a larger proof, such as the one below, but no new constraints are introduced by the larger context.

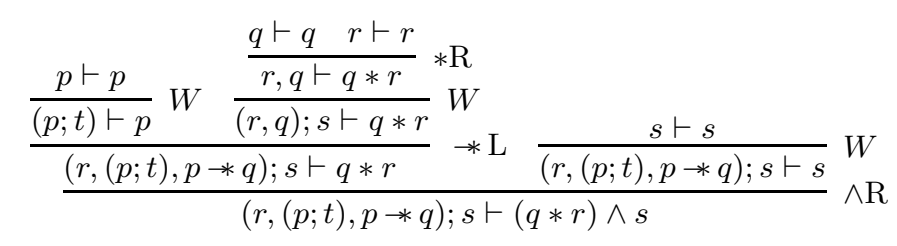

It should be noted that the bunch structure may also simplify some matters. For example, when two variables share the same expression in a multiplicative context, such as the bunch $r[x], s[x]$, it is not hard to see that this is equivalent to $(r, s)[x]$, which is a simpler representation of the same information. It may happen that this latter structure requires a decomposition because of a need to split this multiplicative bunch, in which case we would revert to the former form (and presumably add extra variables to each expression, e.g., $r[x . y], s[x . z]$ ). Thus the bunch structure introduces some further dynamics in the "tagging" of formulæ.

It should also be noted that this tagging is transparent to coherent equivalence. In particular, it should be clear that if $\Gamma \equiv \Delta$, then $\Gamma\left(\Delta^{\prime}[0]\right) \equiv \Delta\left(\Delta^{\prime}[0]\right)$ and $\Gamma\left(\Delta^{\prime}[1]\right) \equiv \Delta\left(\Delta^{\prime}[1]\right)$. It should also be clear that (by abuse of notation) $\Gamma . V(\Delta[0])=\Gamma(\Delta[0]) . V$.

We are now in a position to show the soundness of resource proofs for $\mathbf{B I}$.

Proposition 5 (soundness of resource proofs) Let $\Gamma \vdash \phi$ be a resource sequent in $\mathbf{B I}$. If $\Gamma \vdash \phi$ has a resource proof $R$ with Boolean assignment $I$, then the $\mathbf{L B I}$ proof tree corresponding to $R$ is an $\mathbf{L B I}$ proof of $\Gamma[I]^{1} \vdash \phi$.

Proof We proceed by induction on the size of the resource derivation.

In the base case, the resource proof consists of just one of the leaf rules. Hence there are four cases, of which we only give the argument for Axiom, the others being similar.

In this case, the endsequent of the resource proof is just $\phi\left[e_{1}\right], \Delta \vdash \phi$ with $e_{1}=1$ and $\forall e \in \exp (\Delta) e=0$. Hence $\Gamma[I]^{1}=\phi$ and the LBI proof corresponding to this resource proof is just $\phi \vdash \phi$.

Hence we assume that the result holds for all proofs of no more than a given size.

There are numerous cases, of which we only give the argument for $* \mathrm{~L}, * \mathrm{R}, * \mathrm{~L}, * \mathrm{R}$ and $\rightarrow \mathrm{L}$, the others being similar. 
$* \mathrm{~L}$ : In this case, the endsequent is $\Delta\left(\Gamma, \phi_{1} * \phi_{2}[e]\right) \vdash \phi$ where $e=1$, and the premisses are $\Gamma . V \vdash \phi_{1}$ and $\left.\Delta\left(\Gamma \cdot \bar{V}, \phi_{2}[e]\right) \vdash \phi\right)$. Now as the endsequent has a resource proof, there is a Boolean assignment $I$ of $V$ such that both premisses have resource proofs. Hence by the induction hypothesis we have that the LBI proof tree corresponding to the resource derivation of $\Gamma . V \vdash \phi_{1}$ is an LBI proof of $\Gamma . V[I]^{1} \vdash \phi_{1}$, and the LBI proof tree corresponding to the resource derivation of $\Delta\left(\Gamma \cdot \bar{V}, \phi_{2}[1]\right) \vdash \phi$ is an LBI proof of $\Delta\left(\Gamma . \bar{V}, \phi_{2}[1]\right)[I]^{1} \vdash \phi_{1}$. Now as $\Gamma, \phi_{1} * \phi_{2}$ is the maximal multiplicative super-bunch of $\phi_{1} * \phi_{2}$ in $\Delta$, and as $I$ is a total assignment of Boolean variables, we have that $\Gamma \cdot V[I]^{1}=\Gamma_{1}$ and $\Gamma \cdot \bar{V}[I]^{1}=\Gamma_{2}$ where $\Gamma_{1}, \Gamma_{2}=\Gamma$, and so the $\mathbf{L B I}$ proof tree corresponding to the resource derivation of $\Delta\left(\Gamma, \phi_{1} * \phi_{2}[1]\right) \vdash \phi$ is an LBI proof of $\Delta\left(\Gamma, \phi_{1} * \phi_{2}[1]\right)[I]^{1} \vdash \phi$.

$* \mathrm{R}$ : In this case, the endsequent is $\Gamma \vdash \phi_{1} * \phi_{2}$, and the premiss is $\Gamma, \phi_{1}[1] \vdash \phi_{2}$. Hence by the induction hypothesis we have that the LBI proof tree corresponding to the resource derivation of $\Gamma, \phi_{1}[1] \vdash \phi_{2}$ is an LBI proof of $\Gamma, \phi_{1}[1][I]^{1} \vdash \phi_{2}$, and hence the LBI proof tree corresponding to the resource derivation of $\Gamma \vdash \phi_{1} * \phi_{2}$ is an LBI proof of $\Gamma[I]^{1} \vdash \phi_{1} * \phi_{2}$.

$* \mathrm{~L}$ : In this case, the endsequent is $\Gamma\left(\phi_{1} * \phi_{2}[e]\right) \vdash \phi$ where $e=1$, and the premiss is $\Gamma\left(\phi_{1}[e], \phi_{2}[e]\right) \vdash \phi$. Hence by the induction hypothesis we have that the LBI proof tree corresponding to the resource derivation of $\Gamma\left(\phi_{1}[1], \phi_{2}[1]\right) \vdash \phi$ is an LBI proof of $\Gamma\left(\phi_{1}, \phi_{2}\right)[I]^{1} \vdash \phi$, and hence the LBI proof tree corresponding to the resource derivation of $\Gamma\left(\phi_{1} * \phi_{2}[1]\right) \vdash \phi$ is an LBI proof of $\Gamma\left(\phi_{1} * \phi_{2}\right)[I]^{1} \vdash \phi$.

$* \mathrm{R}$ : In this case, the endsequent is $\Gamma \vdash \phi_{1} * \phi_{2}$, and the premisses are $\Gamma . V \vdash \phi_{1}$ and $\Gamma \cdot \bar{V} \vdash \phi_{2}$, and so there is a Boolean assignment $I$ such that both premisses have a resource-proof. Hence by the induction hypothesis we have that the LBI proof tree corresponding to the resource derivation of $\Gamma . V \vdash \phi_{1}$ is an LBI proof of $\Gamma . V[I]^{1} \vdash \phi_{1}$, and that the $\mathbf{L B I}$ proof tree corresponding to the resource derivation of $\Gamma \cdot \bar{V} \vdash \phi_{2}$ is an $\mathbf{L B I}$ proof of $\Gamma \cdot \bar{V}[I]^{1} \vdash \phi_{2}$. As $I$ is a total assignment of Boolean variables, we have that $\Gamma \cdot V[I]^{1}=\Gamma_{1}$ and $\Gamma \cdot \bar{V}[I]^{1}=\Gamma_{2}$ where $\Gamma_{1}, \Gamma_{2}=\Gamma$, and so the LBI proof tree corresponding to the resource derivation of $\Gamma \vdash \phi_{1} * \phi_{2}$ is an LBI proof of $\Gamma \vdash \phi_{1} * \phi_{2}$.

$\rightarrow \mathrm{L}:$ In this case, the endsequent is $\Gamma\left(\phi_{1} \rightarrow \phi_{2}[e]\right) \vdash \phi$ where $e=1$, and the premisses are $\Gamma \vdash \phi_{1}$ and $\Gamma\left(\phi_{2}[e]\right) \vdash \phi$. Hence by the induction hypothesis we have that the LBI proof tree corresponding to the resource derivation of $\Gamma \vdash \phi_{1}$ is an LBI proof of $\Gamma[I]^{1} \vdash \phi_{1}$, and the LBI proof tree corresponding to the resource derivation of $\Gamma\left(\phi_{2}\right) \vdash \phi$ is an LBI proof of $\Gamma\left(\phi_{2}\right)[I]^{1} \vdash \phi$, and hence the LBI proof tree corresponding to the resource derivation of $\Gamma\left(\phi_{1} \rightarrow \phi_{2}[1]\right) \vdash \phi$ is an LBI proof of $\Gamma\left(\phi_{1} \rightarrow \phi_{2}\right)[I]^{1} \vdash \phi$.

As for MLL and PLL, we require the following lemma:

Lemma 3 Let $\Gamma \vdash \phi$ be a resource sequent in BI. If $\Gamma \vdash \phi$ has a closed resource derivation, then for any bunch $\Delta^{\prime}$ we have that $\Gamma\left(\Delta^{\prime}[0]\right) \vdash \phi$ also has a closed resource derivation.

Proof We proceed by induction on the size of the resource derivation.

In the base case, the resource derivation consists of just one of the leaf rules. Hence there are four cases, of which we only give the argument for Axiom, the others being similar.

In this case, $\Gamma=\phi, \Delta$ and the endsequent of the resource derivation is just $\phi\left[e_{1}\right], \Delta \vdash \phi$ with $e_{1}=1$ and $\forall e \in \exp (\Delta) e=0$. Hence it is clear that there is also a closed resource derivation of $\Gamma\left(\Delta^{\prime}[0]\right) \vdash \phi$.

Hence we assume that the result holds for all proofs of no more than a given size.

There are numerous cases, of which we only give the argument for $* \mathrm{~L}, * \mathrm{R}, * \mathrm{~L}, * \mathrm{R}$ and $\rightarrow \mathrm{L}$, the others being similar.

$* \mathrm{~L}$ : In this case, the endsequent is $\Delta\left(\Gamma, \phi_{1} * \phi_{2}[e]\right) \vdash \phi$ where $e=1$, and the premisses are $\Gamma . V \vdash \phi_{1}$ and $\left.\Delta\left(\Gamma \cdot \bar{V}, \phi_{2}[e]\right) \vdash \phi\right)$. Hence by the hypothesis we have that $\Gamma \cdot V\left(\Delta^{\prime}[0]\right) \vdash \phi_{1}$ and $\left.\Delta\left(\Gamma\left(\Delta^{\prime}[0]\right) . \bar{V}, \phi_{2}[e]\right)\right) \vdash$ $\phi$ ) both have closed resource derivations (recall that the position of the bunch $\Delta^{\prime}$ may be arbitrary), and so $\Delta\left(\Gamma\left(\Delta^{\prime}[0]\right), \phi_{1} * \phi_{2}[e]\right) \vdash \phi$ has a closed resource derivation.

$* \mathrm{R}$ : In this case, the endsequent is $\Gamma \vdash \phi_{1} * \phi_{2}$, and the premiss is $\Gamma, \phi_{1}[e] \vdash \phi_{2}$ where $e=1$. Hence by the induction hypothesis we have that $\Gamma\left(\Delta^{\prime}[0]\right), \phi_{1}[e] \vdash \phi_{2}$ has a closed resource derivation, and hence so does $\Gamma\left(\Delta^{\prime}[0]\right) \vdash \phi_{1} * \phi_{2}$. 
$* \mathrm{~L}$ : In this case, the endsequent is $\Gamma\left(\phi_{1} * \phi_{2}[e]\right) \vdash \phi$ where $e=1$, and the premiss is $\Gamma\left(\phi_{1}[e], \phi_{2}[e]\right) \vdash \phi$. Hence by the induction hypothesis we have that $\Gamma\left(\phi_{1}[e], \phi_{2}[e]\right)\left(\Delta^{\prime}[0]\right) \vdash \phi$ has a closed resource derivation, and hence so does $\Gamma\left(\phi_{1}[e] * \phi_{2}[e]\right)\left(\Delta^{\prime}[0]\right) \vdash \phi$.

$* \mathrm{R}$ : In this case, the endsequent is $\Gamma \vdash \phi_{1} * \phi_{2}$, and the premisses are $\Gamma \cdot V \vdash \phi_{1}$ and $\Gamma \cdot \bar{V} \vdash \phi_{2}$. Hence by the induction hypothesis we have that $\Gamma . V\left(\Delta^{\prime}[0]\right) \vdash \phi_{1}$ and $\Gamma \cdot \bar{V}\left(\Delta^{\prime}[0]\right) \vdash \phi_{2}$ have closed resource derivations, and as $(\Gamma . V)\left(\Delta^{\prime}[0]\right)=\Gamma\left(\Delta^{\prime}[0]\right) . V$, so does $\Gamma\left(\Delta^{\prime}[0]\right) \vdash \phi_{1} * \phi_{2}$.

$\rightarrow \mathrm{L}$ : In this case, the endsequent is $\Gamma\left(\phi_{1} \rightarrow \phi_{2}[e]\right) \vdash \phi$ where $e=1$, and the premisses are $\Gamma \vdash \phi_{1}$ and $\Gamma\left(\phi_{2}[e]\right) \vdash \phi$. Hence by the induction hypothesis we have that $\Gamma\left(\Delta^{\prime}[0]\right) \vdash \phi_{1}$ and $\Gamma\left(\phi_{2}[e]\right)\left(\Delta^{\prime}[0]\right) \vdash \phi$ have closed resource derivations, and hence so does $\Gamma\left(\phi_{1} \rightarrow \phi_{2}[e]\right)\left(\Delta^{\prime}[0]\right) \vdash \phi$.

We are now in a position to show the completeness of resource proofs for $\mathbf{B I}$.

Proposition 6 (completeness of resource proofs) Let $\Gamma \vdash \phi$ be a sequent in BI. If $\Gamma \vdash \phi$ has a proof $\Phi$ in $\mathbf{L B I}$, then there is a set of Boolean variables $V$ such that $\Gamma . V \vdash \phi$ has a resource proof $R$ and the $\mathbf{L B I}$ proof tree corresponding to $R$ is $\Phi$.

Proof We proceed by induction on the height of the LBI proof.

In the base case, the rule used is one of Axiom, $I \mathrm{R}, 1 \mathrm{R}$ and $\perp \mathrm{L}$. We only give the argument for Axiom, the others being similar:

In this case, the sequent is just $\phi \vdash \phi$, and it is clear that this LBI proof corresponds to the resource proof $\overline{\phi[x] \vdash \phi}$ with $x=1$.

Hence we assume that the result holds for all proofs of no more than a given size.

There are numerous cases, of which we only give the argument for $W, * \mathrm{~L}, * \mathrm{R}, * \mathrm{~L}, * \mathrm{R}, \rightarrow \mathrm{L}, \rightarrow \mathrm{R}$, and $\wedge \mathrm{R}$, the others being similar.

W: In this case, the conclusion is $\Gamma\left(\Delta ; \Delta^{\prime}\right) \vdash \phi$ and the premiss is $\Gamma(\Delta) \vdash \phi$, and so by the hypothesis there is a resource proof of $\Gamma(\Delta) . V \vdash \phi$. Now as in such a resource proof all the expressions in $\Gamma(\Delta)$ must be mapped to 1 under the corresponding Boolean assignment, and $\left(\Gamma_{1}, \Gamma_{2}\right)[e]=\Gamma_{1}[e], \Gamma_{2}[e]$, it is clear that there is a resource proof of $\Gamma\left(\Delta ; \Delta^{\prime}\right) . V \vdash \phi$ for which $\Phi$ is the corresponding $\mathbf{L B I}$ proof.

$* \mathrm{~L}$ : In this case, the conclusion is $\Delta\left(\Delta^{\prime}, \Gamma, \phi_{1} * \phi_{2}\right) \vdash \phi$ and the premisses are $\Gamma \vdash \phi_{1}$ and $\Delta^{\prime}\left(\Delta^{\prime}, \phi_{2}\right) \vdash \phi$, and so by the hypothesis there are disjoint sets of variables $V$ and $W$ such that there are resource proofs of $\Gamma . V \vdash \phi_{1}$ and $\Delta\left(\Delta^{\prime}, \phi_{2}\right) . W \vdash \phi$. Now, as these are resource proofs, by Lemma 3 , there exists $V^{\prime}$ such that there are resource proofs of $\left(\Gamma, \Delta^{\prime}\right) \cdot V^{\prime} \vdash \phi_{1}$ and $\Delta\left(\left(\Gamma, \Delta^{\prime}\right) \cdot \overline{V^{\prime}}, \phi_{2}\right) \vdash \phi$. Hence there is a resource proof $R$ of $\Delta\left(\Gamma, \Delta^{\prime}, \phi_{1} * \phi_{2}\right) \vdash \phi$ for which $\Phi$ is the corresponding $\mathbf{L B I}$ proof.

$* \mathrm{R}$ : In this case, the conclusion is $\Gamma \vdash \phi_{1} * \phi_{2}$ and the premiss is $\Gamma, \phi_{1} \vdash \phi_{2}$, and so by the hypothesis there is a resource proof of $\left(\Gamma, \phi_{1}[e]\right) . V \vdash \phi_{2}$, and as $e$ must be 1 , we have that there is a resource proof of $\Gamma . V, \phi_{1}[1] \vdash \phi_{2}$, and hence there is a resource proof $R$ of $\Gamma . V \vdash \phi_{1} * \phi_{2}$ for which $\Phi$ is the corresponding LBI proof.

$* \mathrm{~L}$ : In this case, the conclusion is $\Gamma\left(\phi_{1} * \phi_{2}\right) \vdash \phi$ and the premiss is $\Gamma\left(\phi_{1}, \phi_{2}\right) \vdash \phi$, and so by the hypothesis there is a resource proof of $\Gamma\left(\phi_{1}, \phi_{2}\right) . V \vdash \phi$, and hence there is a resource proof $R$ of $\Gamma\left(\phi_{1} * \phi_{2}\right) . V \vdash \phi$ for which $\Phi$ is the corresponding LBI proof.

$* \mathrm{R}$ : In this case, the conclusion is $\Gamma, \Delta \vdash \phi_{1} * \phi_{2}$, and the premisses are $\Gamma \vdash \phi_{1}$ and $\Delta \vdash \phi_{2}$, and so by the hypothesis there are disjoint sets of variables $V$ and $W$ such that there are resource proofs of $\Gamma . V \vdash \phi_{1}$ and $\Delta . W \vdash \phi_{2}$. Now, as these are resource proofs, by Lemma $\beta$, there exists $V^{\prime}$ such that there are resource proofs of $(\Gamma, \Delta) . V^{\prime} \vdash \phi_{1}$ and $(\Gamma, \Delta) \cdot \overline{V^{\prime}} \vdash \phi_{2}$. Hence there is a resource proof $R$ of $\Gamma, \Delta \vdash \phi_{1} * \phi_{2}$ for which $\Phi$ is the corresponding $\mathbf{L B I}$ proof.

$\rightarrow \mathrm{L}$ : In this case, the conclusion is $\Gamma\left(\phi_{1} \rightarrow \phi_{2}\right) \vdash \phi$ and the premisses are $\Gamma \vdash \phi_{1}$ and $\Gamma\left(\phi_{2}\right) \vdash \phi$, and so by the hypothesis there are resource proofs of $\Gamma . V \vdash \phi_{1}$ and $\Gamma\left(\phi_{2}[e]\right) . W \vdash \phi$. Now $e$ must be 1 under $W$, and as we must have all formulæ in the premisses mapped to 1 , we have that there is a set $V^{\prime}$ of distinct variables $V^{\prime}$ such that there are resource proofs of $\Gamma . V^{\prime} \vdash \phi_{1}$ and $\Gamma\left(\phi_{2}[e]\right) . V^{\prime} \vdash \phi$, and hence there is a resource proof $R$ of $\Gamma\left(\phi_{1} \rightarrow \phi_{2}\right) . V^{\prime} \vdash \phi$ for which $\Phi$ is the corresponding $\mathbf{L B I}$ proof. 
$\rightarrow \mathrm{R}$ : In this case, the conclusion is $\Gamma \vdash \phi_{1} \rightarrow \phi_{2}$ and the premiss is $\Gamma ; \phi_{1} \vdash \phi_{2}$, and so by the hypothesis there is a resource proof of $\left(\Gamma ; \phi_{1}[e]\right) . V \vdash \phi_{2}$, and as $e$ must be 1 , there is a set of distinct variables $V^{\prime}$ such that there is a resource proof of $\Gamma . V^{\prime} ; \phi_{1} \vdash \phi_{2}$, and hence there is a resource proof $R$ of $\Gamma . V^{\prime} \vdash \phi_{1} \rightarrow \phi_{2}$ for which $\Phi$ is the corresponding LBI proof.

$\wedge \mathrm{R}$ : In this case, the conclusion is $\Gamma \vdash \phi_{1} \wedge \phi_{2}$ and the premisses are $\Gamma \vdash \phi_{1}$ and $\Gamma \vdash \phi_{2}$, and so by the hypothesis there are resource proofs of $\Gamma . V \vdash \phi_{1}$ and $\Gamma . W \vdash \phi_{2}$. Now we must have all formulæ in the premisses mapped to 1 , and so we have that there is a set $V^{\prime}$ of distinct variables $V^{\prime}$ such that there are resource proofs of $\Gamma . V^{\prime} \vdash \phi_{1}$ and $\Gamma . V^{\prime} \vdash \phi_{2}$, and hence there is a resource proof $R$ of $\Gamma$. $V^{\prime} \vdash \phi_{1} \wedge \phi_{2}$ for which $\Phi$ is the corresponding $\mathbf{L B I}$ proof.

\section{Strategies}

Hsaving established that the use of Boolean constraints is sound and complete for MLL and two extensions of it, we now turn to the issue of how the constraints generated may be solved. As mentioned above, this is independent of the inference rules themselves.

Clearly there are many different search strategies which could be used to generate a solution to the constraints, but the strategies which we wish to consider here divides conveniently (but not exhaustively) into three: lazy, eager and intermediate. As mentioned above, resource proofs are intended to be independent of a particular strategy, but to contain an explicit specification of the distributive constraints. Strategies are thus distinguished by the manner of solution of the constraints generated during proof-search. We denote as an $n$-strategy one which solves the equations from at most $n$ multiplicative branches at a time.

Lazy distribution. In terms of the calculus introduced above, lazy distribution solves one multiplicative branch's worth of Boolean constraints at a time (thus making it a 1-strategy), and propagates the solution together with any remaining constraints to the next multiplicative branch. This may be thought of as a pessimistic strategy, in that as only a minimal set of constraints is solved, if the derivation turns out to be unsuccessful, then only a minimal amount of work has been done. This strategy is the one most commonly used in linear logic programming languages such as Lygon [4, 17] and Lolli [6], and is analogous to depth-first search.

Eager distribution. The eager distribution is an $\omega$-strategy, in that an unbounded number of equations may be solved, and so all leaves must be closed before any attempt is made to solve the set of constraints. Hence a constraint solver would be called only once per derivation, with a potentially large set of constraints. This may be thought of as an optimistic strategy, in that if one of the branches leads to failure, then the work done on evaluating all the other branches in parallel has been wasted. 9

Note that, analogous to the differences between depth-first and breadth-first search, there are examples in which an eager strategy is preferable to a lazy one. For example, consider the sequent $\Gamma \vdash p \otimes q$ where $\Gamma \vdash p$ is provable and $q$ does not occur anywhere in $\Gamma$ (and hence $\Gamma^{\prime} \forall q$ for any submultiset $\Gamma^{\prime}$ of $\Gamma$ ). Clearly, by an appropriate choice of $\Gamma$ and $p$, the proof of $\Gamma \vdash p$ may be made arbitrarily complex (or, for that matter, infinite if $\Gamma \vdash p$ belongs to an undecidable class of sequents). A lazy strategy will generate the sequents

$$
\frac{\Gamma \vdash p \quad \vdash q}{\Gamma \vdash p \otimes q}
$$

and hence spend an arbitrarily large amount of time on the proof of $\Gamma \vdash p$ (which it will eventually discover is provable) when it is clear that the other branch will fail immediately. However, an eager distribution, which attempts to solve all branches in parallel, will detect that there is no way to close the right-hand branch before any significant amount of work is done, as there is no way to form a putative axiom out of this branch (as $q$ does not appear anywhere in $\Gamma$ ). Hence an eager strategy will be more efficient than a lazy version in this case.

Intermediate distribution. Intermediate strategies are $n$-strategies, where $n \geq 2$, and are analogous to the technique

\footnotetext{
${ }^{6}$ It may also be useful to check that the current constraints have a solution (as distinct from actually solving them), as happens in many constraint logic programming languages.
} 
of iterative deepening. The precise way in which a proof which involves $n+1$ multiplicative branches may be either an eager search for the first $n$ such branches (proceeding from the root), and then lazy searches from then on (effectively performing $n$ lazy searches in parallel), or to "switch" the eager version to a place further from the root (effectively performing a number of lazy searches, one of which is a $n$-way eager search).

As in general it is not possible to predict in advance where the leaves in a proof will be found, it would seem intuitively reasonable to adopt the policy that the eager behaviour occurs towards the root, and once the bound of $n$ is reached, $n$ multiplicative branches are chosen to be explored in a lazy manner. However, this may result in suboptimal behaviour, as the "locality" of the constraints is lost. The alternative would require extra analysis, as initially search would proceed as above, but once the limit is reached, it is necessary to re-assign the $n$ searchers to work on the sub-branches of a particular multiplicative branch in some appropriate way.

For example, consider a 2-strategy with the sequent $p, p, q, q \vdash(p \otimes q) \otimes(p \otimes q)$. It is easy to see that as there are 3 occurrences of $\otimes$ in the formula in the succedent, there will be (at least) 4 multiplicative branches in the ensuing proof. Hence it would be reasonable to use the lazy manner for the first occurrence of $\otimes$, and then solve each generated branch in an eager manner.

Other strategies. The three possibilities of lazy, eager and intermediate are probably the most natural choices of strategies, but they are not the only ones. One such strategy (which may be thought of as a variation of a purely lazy strategy) may be described as "fact-first", in that given a sequent such as $\Gamma \vdash p \otimes q$, we can note that if $q \in \Gamma$ then one of the multiplicative branches will form an axiom. Hence, rather than arbitrarily selecting one of the branches (the purely lazy strategy), we select the putative axiom, generate the appropriate constraints, and continue with the other branch. Thus the strategy is adaptive, in that the order of evaluation will depend on the sequents generated.

Examples. Consider the MLL sequent $p, p, q, q \vdash(p \otimes q) \otimes(p \otimes q)$.

A resource proof of this sequent is of the form

$$
\frac{\frac{P_{1}}{p\left[x_{1}\right], p\left[x_{2}\right], q\left[x_{3}\right], q\left[x_{4}\right] \vdash p \otimes q} \quad \frac{P_{3}}{p\left[\overline{x_{1}}\right], p\left[\overline{x_{2}}\right], q\left[\overline{x_{3}}\right], q\left[\overline{x_{4}}\right] \vdash p \otimes q}}{p, p, q, q \vdash(p \otimes q) \otimes(p \otimes q)},
$$

where the leaves are as follows:

$$
\begin{array}{ll}
P_{1}: p\left[x_{1} \cdot y_{1}\right], p\left[x_{2} \cdot y_{2}\right], q\left[x_{3} \cdot y_{3}\right], q\left[x_{4} \cdot y_{4}\right] \vdash p & P_{3}: p\left[\overline{x_{1}} \cdot z_{1}\right], p\left[\overline{x_{2}} \cdot z_{2}\right], q\left[\overline{x_{3}} \cdot z_{3}\right], q\left[\overline{x_{4}} \cdot z_{4}\right] \vdash p \\
P_{2}: p\left[x_{1} \cdot \overline{y_{1}}\right], p\left[x_{2} \cdot \overline{y_{2}}\right], q\left[x_{3} \cdot \overline{y_{3}}\right], q\left[x_{4} \cdot \overline{y_{4}}\right] \vdash q & P_{4}: p\left[\overline{x_{1}} \cdot \overline{z_{1}}\right], p\left[\overline{x_{2}} \cdot \overline{z_{2}}\right], q\left[\overline{x_{3}} \cdot \overline{z_{3}}\right], q\left[\overline{x_{4}} \cdot \bar{z}_{4}\right] \vdash q
\end{array}
$$

The lazy strategy yields the following sequence of constraints and solutions:

\begin{tabular}{|c|c|c|}
\hline Leaf & Constraints added & Solutions \\
\hline \hline$P_{1}$ & $x_{1} \cdot y_{1}=1, x_{2} \cdot y_{2}=0, x_{3} \cdot y_{3}=0, x_{4} \cdot y_{4}=0$ & $x_{1}=1, y_{1}=1$ \\
\hline$P_{2}$ & $x_{2} \cdot \overline{y_{2}}=0, x_{3} \cdot \overline{y_{3}}=1, x_{4} \cdot \overline{y_{4}}=0$ & $x_{3}=1, y_{3}=0, x_{2}=0, x_{4}=0$ \\
\hline$P_{3}$ & $z_{2}=1, z_{4}=0$ & $z_{2}=1, z_{4}=0$ \\
\hline$P_{4}$ & $\overline{x_{4}} \cdot \overline{z_{4}}=1$ & \\
\hline
\end{tabular}

which gives us the overall solution

$$
\begin{array}{r}
\mathrm{x}_{1}=1, \mathrm{x}_{2}=0, \mathrm{x}_{3}=1, \mathrm{x}_{4}=0, \\
\mathrm{y}_{1}=1, \mathrm{y}_{2}=0, \mathrm{y}_{3}=0, \mathrm{y}_{4}=0, \\
\mathrm{z}_{1}=0, \mathrm{z}_{2}=1, \mathrm{z}_{3}=0, \mathrm{z}_{4}=\mathbf{0}
\end{array}
$$

where $y_{2}, y_{4}, z_{1}$ and $z_{3}$ have been arbitrarily assigned the value 0 . Note that we can conclude from the equations $x_{4} \cdot y_{4}=0$ and $x_{4} \cdot \overline{y_{4}}=0$ that $x_{4}$ must be 0 , and similarly for $x_{2}$.

The eager strategy collects the entire set of equations below, and then solves it to produce the same overall solution.

$$
\begin{array}{ll}
\mathrm{x}_{1} \cdot \mathrm{y}_{1}=1, \mathrm{x}_{2} \cdot \mathrm{y}_{2}=0, \mathrm{x}_{3} \cdot \mathrm{y}_{3}=0, \mathrm{x}_{4} \cdot \mathrm{y}_{4}=0 & \overline{\mathrm{x}_{1}} \cdot \mathrm{z}_{1}=0, \overline{\mathrm{x}_{2}} \cdot \mathrm{z}_{2}=1, \overline{\mathrm{x}_{3}} \cdot \mathrm{z}_{3}=0, \overline{\mathrm{x}_{4}} \cdot \mathrm{z}_{4}=0 \\
\mathrm{x}_{1} \cdot \overline{\mathrm{y}_{1}}=\mathbf{0}, \mathrm{x}_{2} \cdot \overline{\mathrm{y}_{2}}=\mathbf{0}, \mathrm{x}_{3} \cdot \overline{\mathrm{y}_{3}}=1, \mathrm{x}_{4} \cdot \overline{\mathrm{y}_{4}}=\mathbf{0} & \overline{\mathrm{x}_{1}} \cdot \overline{\mathrm{z}_{1}}=\mathbf{0}, \overline{\mathrm{x}_{2}} \cdot \overline{\mathrm{z}_{2}}=\mathbf{0}, \overline{\mathrm{x}_{3}} \cdot \overline{\mathrm{z}_{3}}=\mathbf{0}, \overline{\mathrm{x}_{4}} \cdot \overline{\mathrm{z}_{4}}=1
\end{array}
$$

One variant of the intermediate strategy first solves the equations for $P_{1}$ and $P_{3}$ in parallel, and then those for $P_{2}$ and $P_{4}$ : 


\begin{tabular}{|c|c|c|}
\hline Leaf & Constraints added & Solutions \\
\hline \hline$P_{1}, P_{3}$ & $x_{1} \cdot y_{1}=1, x_{2} \cdot y_{2}=0, x_{3} \cdot y_{3}=0, x_{4} \cdot y_{4}=0$ & $x_{1}=1, y_{1}=1$ \\
\hline & $\overline{x_{1}} \cdot z_{1}=0, \overline{x_{2}} \cdot z_{2}=1, \overline{x_{3}} \cdot z_{3}=0, \overline{x_{4}} \cdot z_{4}=0$ & $x_{2}=0, z_{2}=1$ \\
\hline$P_{2}, P_{4}$ & $x_{2} \cdot \overline{y_{2}}=0, x_{3} \cdot \overline{y_{3}}=1, x_{4} \cdot \overline{y_{4}}=0$ & $x_{3}=1, y_{3}=0$ \\
\hline & $\overline{x_{3}} \cdot \overline{z_{3}}=0, \overline{x_{4}} \cdot \overline{z_{4}}=1$ & $x_{4}=0, z_{4}=0$ \\
\hline
\end{tabular}

The other variant of the intermediate strategy first solves the equations for $P_{1}$ and $P_{2}$ in parallel, and then those for $P_{3}$ and $P_{4}$ :

\begin{tabular}{|c|c|c|}
\hline Leaf & Constraints added & Solutions \\
\hline \hline$P_{1}, P_{2}$ & $x_{1} \cdot y_{1}=1, x_{2} \cdot y_{2}=0, x_{3} \cdot y_{3}=0, x_{4} \cdot y_{4}=0$ & $x_{1}=1, y_{1}=1$ \\
\hline & $x_{1} \cdot \overline{y_{1}}=0, x_{2} \cdot \overline{y_{2}}=0, x_{3} \cdot \overline{y_{3}}=1, x_{4} \cdot \overline{y_{4}}=0$ & $x_{3}=1, y_{3}=0, x_{4}=0, x_{2}=1$ \\
\hline$P_{3}, P_{4}$ & $z_{2}=1, z_{4}=0$ & $z_{2}=1, z_{4}=0$ \\
\hline & $z_{2}=1, \overline{z_{4}}=1$ & \\
\hline
\end{tabular}

Note that solving the equations for $P_{1}$ and $P_{2}$ in parallel generates more of the solution than solving those for $P_{1}$ and $P_{3}$ in parallel.

Consider now the PLL sequent $p, q, q \vdash(p \otimes q) \oplus(p \otimes q \otimes q)$.

This has the following resource derivation.

$$
\frac{\frac{P_{1}}{p\left[y_{1}\right], q\left[y_{2}\right], q\left[y_{3}\right] \vdash(p \otimes q)[0], p \otimes q} \quad p\left[\overline{y_{1}}\right], q\left[\overline{y_{2}}\right], q\left[\overline{y_{3}}\right] \vdash(p \otimes q)[0], q}{\frac{p, q, q \vdash(p \otimes q)[x],(p \otimes q \otimes q)[\bar{x}]}{p, q, q \vdash(p \otimes q) \oplus(p \otimes q \otimes q)} \oplus \mathrm{R}} \otimes \mathrm{R}
$$

where $P_{1}$ and $P_{2}$ are as follows:

$$
\begin{aligned}
& P_{1}: p\left[y_{1} \cdot z_{1}\right], q\left[y_{2} . z_{2}\right], q\left[y_{3} . z_{3}\right] \vdash(p \otimes q)[0], p \\
& P_{2}: p\left[y_{1} \cdot \overline{z_{1}}\right], q\left[y_{2} \cdot \overline{z_{2}}\right], q\left[y_{3} . \overline{z_{3}}\right] \vdash(p \otimes q)[0], q
\end{aligned}
$$

Solving the equations gives us

$$
x=0, y_{1}=1, y_{2}=1, y_{3}=0, z_{1}=1, z_{2}=0, z_{3}=0
$$

Note that $x=0$ because of the choice of principal formula in the application of the $\oplus \mathrm{R}$ rule.

This gives us the resource proof

$$
\begin{aligned}
& \underline{p[1], q[0], q[0] \vdash(p \otimes q)[0], p \quad p[0], q[1], q[0] \vdash(p \otimes q)[0], q}
\end{aligned}
$$

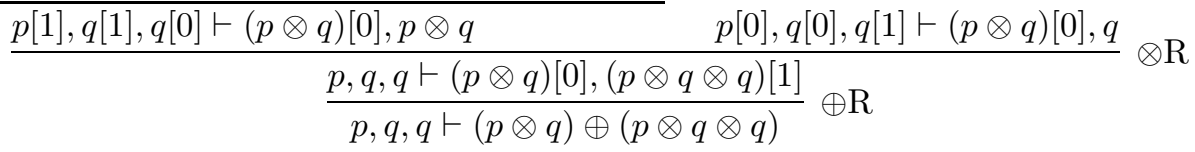

and hence the sequent proof

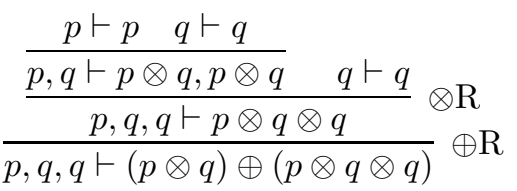

Note that, in this case, the various strategies will only differ on the sub-proof $p, q, q \vdash p \otimes q \otimes q$ in the manner discussed above.

Turning to an example of an unprovable sequent, consider

$$
p \otimes q, r \vdash p \otimes q
$$

The only possible resource derivation will look like this: 


$$
\frac{p\left[x_{1}\right], q\left[x_{2}\right], r\left[x_{3}\right] \vdash p \quad p\left[\overline{x_{1}}\right], q\left[\overline{x_{2}}\right], r\left[\overline{x_{3}}\right] \vdash q}{\frac{p, q, r \vdash p \otimes q}{p \otimes q, r \vdash p \otimes q} \otimes \mathrm{L}}
$$

The lazy strategy would solve, say, the left-hand leaf first, resulting in the equations

$$
x_{1}=1, x_{2}=0, x_{3}=0
$$

which would make the second leaf into

$$
p[0], q[1], r[1] \vdash q
$$

which clearly cannot be made into an axiom, and the search fails at this point.

The eager strategy would generate the equations

$$
x_{1}=1, x_{2}=0, x_{3}=0
$$

from the left-hand leaf, together with the equations

$$
x_{1}=1, x_{2}=0, x_{3}=1
$$

from the second leaf, and hence determine that the union of these two sets of equations has no solution. Turing to an example from $\mathbf{B I}$, consider the sequent

$$
(r,(p ; t), p * q) ; s \vdash q * r
$$

This results in the following resource derivation:

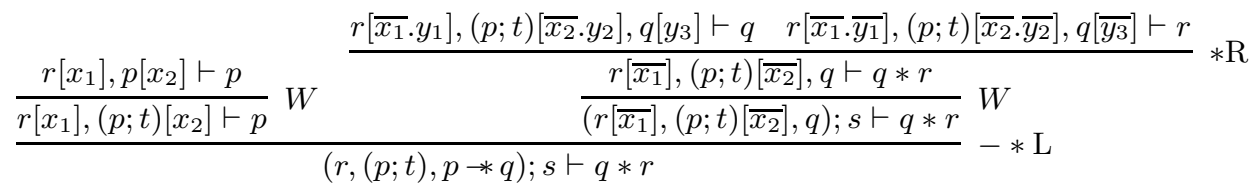

Note that the fact that $(r,(p ; t), p * q) ; s$ has an outermost additive context means that the distribution problem for the $* \mathrm{~L}$ rule is to determine how to distribute the multiplicative sub-context $r,(p ; t)$.

A lazy evaluation using the leftmost leaf first will give us

$$
x_{1}=0, x_{2}=1
$$

Propagating this to the next leaf will give us

$$
r\left[y_{1}\right],(p ; t)[0], q\left[y_{3}\right] \vdash q
$$

from which we determine $y_{1}=0, y_{3}=1$. Passing this onto the third leaf gives us

$$
r[1],(p ; t)[0], q[0] \vdash r
$$

as required.

An eager evaluation would generate the set of equations below.

$$
x_{1}=0, x_{2}=1, \overline{x_{1}} \cdot y_{1}=0, \overline{x_{2}} \cdot y_{2}=0, y_{3}=1, \overline{x_{1}} \cdot \overline{y_{1}}=1, \overline{x_{2}} \cdot \overline{y_{2}}=0, \overline{y_{3}}=0
$$

Solving this set of equations gives

$$
x_{1}=0, x_{2}=1, y_{1}=0, y_{2}=, y_{3}=1
$$

as above. 


\section{The generality of the method}

We have seen that the method of calculating the distribution of formulæ during proof-search with multiplicative rules via solving systems of Boolean constraint equations may be applied to both linear logic and BI. Thus these two systems illustrate the use of our methods for the two systems' different treatments of Weakening and Contraction, and for the relationship between linear and intuitionistic implication, based both on exponentials and on bunching.

Whilst BI can be seen as the free combination of its linear and intuitionistic fragments, the family of "traditional" relevant logics, as represented in [12, 13, make choices of connectives and their associated laws which, at least from a semantic perspective, are very much more ad hoc.

However, Read's taxonomy [12] provides a setting for a systematic assessment of the applicability of our methods. Read identifies a basic substructural system, DW, and then considers a zoo of possible extensions. We conjecture that our method is sufficiently general to deal with essentially any system involving multiplicative rules. Below we briefly consider the systematic classification of a large family of systems of relevant logic presented by Read [12].

The basic system, DW, is formulated as a bunched natural deduction system [12, 11]. The collection of connectives is essentially the same as that taken in $\mathbf{B I}$ and the natural deduction system is, of course, closely related to LBI. The introduction rules are the same as the right rules of LBI. The elimination rules stand in the usual relationship to the left rules of LBI. For example, the elimination rules for the multiplicative (intensional) conjunction are implication are, respectively, []

$$
\otimes \mathrm{E} \quad \frac{\Gamma \vdash \phi \otimes \phi \quad \Delta(\phi, \psi) \vdash \chi}{\Delta(\Gamma) \vdash \chi} \text { and } \multimap \mathrm{E} \quad \frac{\Gamma \vdash \phi \multimap \psi \quad \Delta \vdash \phi}{\Gamma, \Delta \vdash \psi} .
$$

The exceptions are the rules for (a classical) negation. The rules for negation are, in our notation,

$$
\sim \mathrm{I} \frac{\Gamma, \phi \vdash \sim \psi \Delta \vdash \phi}{\Gamma, \Delta \vdash \sim \phi} \quad \text { and } \quad \sim \mathrm{E} \frac{\Gamma \vdash \sim \phi}{\Gamma \vdash \phi},
$$

with the optional additional rule,

$$
\mathrm{CM} \frac{\Gamma, \phi \vdash \sim \phi}{\Gamma \vdash \sim \phi} \quad \text { "consequentia mirabilis". }
$$

The structural rules of DW are:

- The monoid (identity, commutativity, associativity) laws for additive bunching;

- Weakening and Contraction for additive bunching;

- A left-identity for multiplicative bunching, $\emptyset_{m}, \Gamma \equiv \Gamma$.

Note that the basic system DW does not assume commutativity of ",".

To describe the various additional structural properties which a system, we write $\Gamma \leq \Gamma^{\prime}$, for bunches $\Gamma, \Gamma^{\prime}$ to denote that $\Delta(\Gamma) \vdash \phi$ implies $\Delta\left(\Gamma^{\prime}\right) \vdash \phi$. The family of relevant logics can then be presented systematically as a hierarchy of systems regulated by the axioms given, using Read's terminology but, as above, our notation, in Table 1 .

We can now define, following [12], the following systems:

$$
\begin{aligned}
& \mathbf{T W}=\mathrm{B}+\mathrm{B}^{\prime} \quad \mathbf{D L}=\mathbf{D W}+\mathrm{CM}+\mathrm{WB} \\
& \mathbf{R W}=\mathbf{T W}+\mathbf{C} \quad \mathbf{T} \mathbf{L}=\mathbf{T W}+\mathbf{C M}+\mathbf{W B}\left(=\mathbf{D L}+\mathrm{B}+\mathrm{B}^{\prime}\right) \\
& \mathbf{T}=\mathbf{T L}+\mathrm{W} \quad \mathbf{E}=\mathbf{T}+\mathrm{C}^{* *} \\
& \mathbf{R}=\mathbf{E}+\mathrm{C}^{*} \quad(=\mathbf{R W}+\mathrm{W}) \quad \mathbf{R M}=\mathbf{R}+\mathrm{K}^{*}
\end{aligned}
$$

BI is the system which takes just identity, associativity and commutativity for ",".

The system DW, and so each of the systems defined above, supports the distributive law for additive conjunction and disjunction:

$$
\phi \wedge(\psi \vee \chi) \dashv(\phi \wedge \psi) \vee(\phi \wedge \chi)
$$

\footnotetext{
${ }^{7}$ We use a natural deduction system here in order to conveniently characterize the a collection of relevant systems. However, the failure of the subformula property for rules such as $\multimap$ E makes effective search problematic.

${ }^{8}$ We use "," for multiplicative bunching and ";" for additive bunching; Read's notation is the exact opposite. We write $\emptyset_{m}$ for the multiplicative identity; Read uses t.
} 


\begin{tabular}{|c|c|c|}
\hline B & $\Gamma,(\Delta, \Theta) \leq(\Gamma, \Delta), \Theta$ & prefixing \\
\hline $\mathrm{B}^{\prime}$ & $\Gamma,(\Delta, \Theta) \leq(\Delta, \Gamma), \Theta$ & suffixing \\
\hline $\mathrm{C}^{* *}$ & $\Gamma, \emptyset_{m} \leq \Gamma$ & right-identity \\
\hline $\mathrm{C}^{*}$ & $\Gamma, \Delta \leq \Delta, \Gamma$ & assertion \\
\hline $\mathrm{C}$ & $(\Gamma, \Delta), \Theta \leq(\Gamma, \Theta), \Delta$ & permutation \\
\hline $\mathrm{W}^{*}$ & $\Gamma, \Gamma \leq \Gamma$ & conjunctive assertion \\
\hline W & $(\Gamma, \Delta), \Delta$ & contraction \\
\hline WB & $\Gamma,(\Gamma, \Delta) \leq \Gamma, \Delta$ & conjunctive syllogism \\
\hline $\mathrm{S}^{*}$ & $\Delta,(\Gamma, \Delta) \leq \Gamma, \Delta$ & \\
\hline S & $(\Gamma, \Theta),(\Delta, \Theta) \leq(\Gamma, \Delta), \Theta$ & \\
\hline $\mathrm{K}^{*}$ & $\Gamma \leq \Gamma, \Gamma$ & mingle / premiss repetition \\
\hline $\mathrm{K}$ & $\Gamma \leq \Gamma, \Delta$ & affinity \\
\hline
\end{tabular}

Table 1: Relevant Axioms

In this sense, the family of relevant systems is closer to BI than to linear logic. Indeed, linear logic without the exponentials may be seen, relative to this hierarchy, as sitting below even DW, the rules for its additives not even permitting distribution. Classical logic arises as $\mathbf{R}+\mathrm{K}$, so that we recover a connection, via the exponentials, with the non-distributive, linear systems.

Turning at last to resource proofs, we can make the following observations:

- The natural deduction-style operational rules of DW are, apart from negation, all handled just as for their counterparts in LBI, the introduction rules being the same as the right rules and the elimination rules typically being simpler than the corresponding left rules. For example, the resource versions of the $\otimes \mathrm{E}$ and $\multimap \mathrm{E}$ rules may be formulated as, respectively,

$$
\otimes \mathrm{E} \quad \frac{\Gamma \cdot V \vdash \phi \otimes \phi \quad \Delta(\Gamma \cdot \bar{V}, \phi, \psi) \vdash \chi}{\Delta(\Gamma) \vdash \chi} \text { and } \multimap \mathrm{E} \quad \frac{\Gamma \cdot V \vdash \phi \multimap \psi \quad \Gamma \cdot \bar{V} \vdash \phi}{\Gamma \vdash \psi} .
$$

The other operational rules, excluding negation, are treated similarly;

- The $\sim$ E rule, being unary with only right-hand activity, is trivial. The resource version of the $\sim$ I rule is

$$
\sim I \quad \frac{\Gamma \cdot V, \phi[e] \vdash \sim \psi \quad \Gamma \cdot \bar{V} \vdash \phi}{\Gamma \vdash \sim \phi} \quad e=1 .
$$

The CM rule goes as

$$
\mathrm{CM} \frac{\Gamma, \phi[e] \vdash \sim \phi}{\Gamma \vdash \sim \phi} \quad e=1 ;
$$

- The various structural rules can now be seen to be unproblematic. For example, $\mathrm{W}^{*}, \mathrm{~W}, \mathrm{WB}, \mathrm{S}^{*}$ and $\mathrm{S}$ all follow the pattern of Contraction in BI. Similarly, $\mathrm{K}^{*}$ and $\mathrm{K}$ follow the pattern of Weakening in BI.

Our usual soundness and completeness properties can now established for the family of relevant systems .

Thus our analysis encompasses the full range of established (propositional) substructural logics. We conjecture that our methods can be applied to the multiplicative (or intensional) quantifiers introduced in [8, 10] and developed in [11]. The usual additive (or extensional) quantifiers were treated in [5] (they are unproblematic).

Acknowledgements. We thank John Crossley, Samin Ishtiaq, Michael Winikoff and anonymous referees from CADE14 for their comments on this work. Partial support from the British Council, the UK EPSRC and the RMIT Faculty of Applied Science is gratefully acknowledged. Harland is grateful for the hospitality of the Department of Computer Science of Queen Mary and Westfield College, University of London during a period of sabbatical leave. Pym is grateful for the partial support of the EPSRC. 


\section{References}

[1] D. Gabbay, Labelled Deductive Systems: Principles and Applications, Vol. 1: Basic Principles, Oxford University Press, 1996.

[2] J.-Y. Girard, Linear Logic, Theoretical Computer Science 50, 1-102, 1987.

[3] J.-Y. Girard, Proof-nets for Additives, Manuscript, 1994.

[4] J. Harland, D. Pym and M. Winikoff, Programming in Lygon: An Overview, Proceedings of the International Conference on Algebraic Methodology and Software Technology (AMAST) 391-405, M. Wirsing and M. Nivat (eds.), July, 1996. Published by Springer-Verlag as Lecture Notes in Computer Science 1101.

[5] J. Harland and D. Pym, Resource-distribution via Boolean constraints (extended abstract), Proceedings of the International Conference on Automated Deduction (CADE-14) 222-236, W. McCune (ed.), Townsville, July, 1997. Published by Springer-Verlag as Lecture Notes in Computer Science 1249.

[6] J. Hodas and D. Miller, Logic Programming in a Fragment of Intuitionistic Linear Logic, Information and Computation 110:2:327-365, 1994.

[7] S. Kripke, Semantical Analysis of Intuitionistic Logic, I. In J.N. Crossley and M.A.E. Dummett, editors, Formal Systems and Recursive Functions, 92-130. North-Holland, Amsterdam, 1965.

[8] P. O'Hearn and D. Pym, The Logic of Bunched Implications, Bulletin of Symbolic Logic 5(2), June 1999, 215-244.

[9] P. O'Hearn, D. Pym and H. Yang, Possible worlds and resources: the semantics of BI. Manuscript, submitted, 2000. Available at http://www.dcs.qmw.ac.uk/ pym.

[10] D. Pym, On Bunched Predicate Logic, Proceedings of the 14th IEEE Symposium on Logic in Computer Science, 183-192, Trento, Italy, July, 1999. IEEE Computer Society, 1999.

[11] D. Pym, The Semantics and Proof Theory of the Logic of Bunched Implications, forthcoming monograph, 2000.

[12] S. Read, Relevant Logic, Blackwell, 1988.

[13] G. Restall, An Introduction to Substructural Logics, Routledge, 2000.

[14] D. Pym and J. Harland, A Uniform Proof-theoretic Investigation of Linear Logic Programming, Journal of Logic and Computation 4:2:175-207, April, 1994.

[15] T. Tammet, Proof Search Strategies in Linear Logic, Journal of Automated Reasoning 12:273-304, 1994.

[16] A. Urquhart. Semantics for Relevant Logics, Journal of Symbolic Logic 37, 159-169, 1972.

[17] M. Winikoff and J. Harland, Implementing the Linear Logic Programming Language Lygon, in Proceedings of the International Logic Programming Symposium 66-80, J. Lloyd (ed.), Portland, December, 1995. Published by MIT Press. 